\title{
Identification of selected sources from the ROSAT Galactic Plane Survey. I. ${ }^{\star \star \star \star}$
}

\author{
C. Motch $^{1}$, P. Guillout ${ }^{2,1}$, F. Haberl ${ }^{2}$, J. Krautter ${ }^{3}$, M.W. Pakull ${ }^{1}$, W. Pietsch ${ }^{2}$, K. Reinsch ${ }^{4}$, W. Voges ${ }^{2}$, and \\ F.-J. Zickgraf ${ }^{1}$ \\ 1 Observatoire Astronomique, UMR 7550 CNRS, 11 rue de l'Université, F-67000 Strasbourg, France \\ 2 Max-Planck-Institut für extraterrestrische Physik, D-85740, Garching bei München, Germany \\ 3 Landessternwarte, D-69117 Heidelberg-Königstuhl, Germany \\ 4 Universitäts-Sternwarte, Geismarlandstrasse 11, D-37083, Göttingen, Germany
}

Received December 9, 1997; accepted April 30, 1998

\begin{abstract}
We report on optical searches in the error circles of 93 ROSAT survey sources located at low galactic latitudes $\left(|b|<20^{\circ}\right)$. These sources were extracted from the ROSAT Galactic Plane Survey using various selection criteria on hardness ratio, X-ray and optical brightness and integrated galactic absorption in the direction of the source. We find optical identifications in 76 cases, among which are 25 new AGN, 6 new CVs and a new Be/X-ray binary. In order to illustrate the relevance of the source selections applied here, we cross-correlated the ROSAT all-sky survey bright source list with SIMBAD. Different classes of X-ray emitters populate distinct regions of a multi dimensional parameter space involving flux ratios, galactic latitude and $N_{\mathrm{H}}$. This relatively good segregation offers the possibility to build source samples with enhanced probability of identification with a given class. Complete optical identification of such subsamples could eventually be used to compute meaningful probabilities of identification for all sources using as basis a restricted set of multi-wavelength information.
\end{abstract}

Key words: X-ray general - X-ray stars - X-rays: galaxies - surveys

Send offprint requests to: C. Motch

* Partly based on observations obtained at the European Southern Observatory, La Silla (Chile) with the $2.2 \mathrm{~m}$ telescope of the Max-Planck-Society, with the ESO $1.5 \mathrm{~m}$ telescope and at the Observatoire de Haute-Provence, CNRS, France.

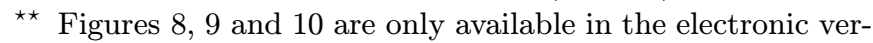
sion. Tables 4 and 5 are also available in electronic form at the CDS via anonymous ftp to cdsarc.u-strasbg.fr (130.79.128.5) or via http://cdsweb.u-strasbg.fr/Abstract.html

\section{Introduction}

The ROSAT all-sky survey (RASS; Voges 1992) was performed from 1990 July till 1991 February and was carried out with the X-ray telescope (XRT) and the Position Sensitive Proportional Counter (PSPC, Pfeffermann et al. 1986). The survey mapped as many as 60000 new sources in the soft X-ray band $(0.1-2.4 \mathrm{keV}$, Maximum Likelihood, $\mathrm{ML} \geq 10$ ) down to limiting fluxes of the order of a few $10^{-13} \mathrm{erg} \mathrm{cm}^{-2} \mathrm{~s}^{-1}$. At this detection threshold, there are about $1.4510^{-2}$ spurious sources per square degree. Recently, the 18811 RASS sources having a PSPC count rate larger than $0.05 \mathrm{cts} \mathrm{s}^{-1}$ and $\mathrm{ML} \geq 15$ have been published (Voges et al. 1996). The study of the RASS point source content at low galactic latitudes $\left(|b| \leq 20^{\circ}\right)$ is the scope of a dedicated project, the ROSAT Galactic Plane Survey (RGPS: Motch et al. 1991b). About 15000 RASS sources are located in this $40^{\circ}$ wide strip of the sky.

In the galactic plane area, already $20 \%$ to $30 \%$ of RGPS sources may be identified with high confidence on the basis of positional coincidence with objects, mostly stars, catalogued in the SIMBAD database. Adding crosscorrelation with relatively bright stellar catalogues such as Guide Star Catalogue entries allows the identification of about $50 \%$ of RGPS sources (Motch et al. 1997a).

The number of remaining unidentified sources is however still too large to allow their systematic identification at the telescope. In order to tackle this problem, two paths of investigations were chosen; i) selection of sources over the whole galactic sky using criteria on their X-ray characteristics and optical content of the error box and ii) selection of sample areas at judicious positions for complete optical identification.

The X-ray selected approach has led to the discovery of a number of interesting objects, mostly soft sources which have escaped previous surveys carried out at higher 
energies and furthermore without imaging instrumentation or large sky coverage. Among the most noticeable results are the discovery of galactic supersoft sources (Ögelman et al. 1993; Motch et al. 1994; Beuermann et al. 1995), a new class of soft intermediate polars (Haberl \& Motch 1995), and more recently the identification of a couple of isolated neutron stars presumably accreting from the interstellar medium (Walter et al. 1996; Haberl et al. 1997).

On the other hand, systematic optical identification of RGPS sources in sample areas has led to the conclusion that active coronae dominate by number the point source content in the galactic plane strip (up to $85 \%$ of the total; Motch et al. 1997a). In contrast, the stellar fraction identified at high galactic latitude is much lower $(12-63 \%$; Zickgraf et al. 1997). The modelling of this stellar population and ages derived from Lithium lines clearly show that the stars detected in the RASS are quite young, mostly younger than 1 Gyr (Guillout et al. 1996a,b). Another important result from this study is derived from the small number of sources remaining unidentified. In particular, this implies that the space density of isolated neutron stars accreting from the interstellar medium is at least a factor 10 smaller than expected.

This paper is divided in two parts. First we show how $\mathrm{X}$-ray and optical characteristics of ROSAT sources may be used to build samples with enhanced probabilities of identification with a given class of object. For that purpose we cross-identified the ROSAT all sky survey bright source list with SIMBAD. Second, we report on optical observations of 93 selected RGPS sources. The source list contains various kinds of X-ray selected sub samples out of which several identifications not repeated here were already published.

Our identification programme of X-ray selected sources in the galactic plane is continuing and we expect to provide more RGPS source identifications in a future paper.

\section{Cross-identification of the ROSAT all-sky survey bright source catalogue with SIMBAD}

\subsection{BRASS-SIMBAD correlation}

Studying the clustering properties of identified X-ray sources in a multi-wavelength parameter space can help finding domains in which the probability of association with a given class of object is higher than on the average. For instance, at low galactic latitude the overwhelming domination of stellar coronae makes the discovery of new $\mathrm{CVs}$ a difficult task as they only account for $\approx 1 \%$ of the total number of sources (Motch et al. 1996) whereas their frequency is much higher among either hard, or soft and bright selected sources.

If completely identified flux limited samples are obtained using catalogue cross-correlation or work at the telescope, then the probability that X-ray emission from a given source originates from a particular class of emitters (star, AGN, etc.) may be eventually estimated in a quantitative manner. This allows the computation of a statistical identification using as input the restricted set of multi-wavelength parameters available for all sources.

RGPS sources identified in SIMBAD are far from making a complete sample. SIMBAD is roughly optical flux limited, although at very different levels depending on the nature, stellar or extragalactic, of the object. As most of the recent additions are coming from the literature, the whole collection is highly heterogeneous. Clearly, the set of SIMBAD identifications cannot be used to provide real probabilities of identification. However, it may be used to construct selected samples of presumably enhanced probabilities.

A first analysis was made in 1991, using X-ray and optical characteristics of a limited number of stars, AGN, CVs and accreting binaries identified in preliminary and partial releases of the RASS (Motch 1991a). The selection criteria used to build the source samples studied in this paper are derived from this early analysis.

The recently published ROSAT all-sky survey bright source catalogue (BRASS) by Voges et al. (1996) constitutes a sample of larger size and of much better quality than the one originally used in 1991. Therefore, for the sole aim of illustrating in an extensive manner the X-ray selection criteria used in this work, we decided to crosscorrelate BRASS with SIMBAD. We underline that the associations between bright RASS sources and SIMBAD entries used in this paper have not been human screened and cannot be considered individually as real identifications. Nevertheless their overall statistical properties may still be used for our purpose.

The BRASS covers the whole sky whereas the RGPS is by definition limited to the galactic plane area. However, selection rules arising from the BRASS catalogue can usefully be applied to the restricted RGPS area. The variability of parameters with galactic latitude or $N_{\mathrm{H}}$ can also be better studied using the whole sky catalogue.

The first step of the cross-correlation consisted in extracting from SIMBAD all objects located within $3^{\prime}$ from the ROSAT source, retrieving the optical position and associated error, the first identifier, possible ROSAT name, optical magnitudes, spectral types, full object type, number of references and Einstein measurements when available. In a second step, we retained from the SIMBAD cross correlation log entries having a non null intersection between the ROSAT 90\% confidence circle and the SIMBAD error circle. As the error radius of Einstein sources is underestimated in SIMBAD, we updated it manually to $50^{\prime \prime}$. When several SIMBAD objects remained, we retained the one closest to the X-ray position. With a mean $90 \%$ error radius of $27^{\prime \prime}$ for BRASS sources, and a total number of $\sim 1.4610^{6}$ SIMBAD objects having in most cases small positional errors $\left(\leq 1^{\prime \prime}\right)$, we expect about 120 
Table 1. BRASS-SIMBAD correlation

\begin{tabular}{lrr}
\hline Sample & Number & $\begin{array}{r}\text { fraction of } \\
\text { Total BRASS (\%) }\end{array}$ \\
\hline Unidentified & 10705 & 56.9 \\
1 SIMBAD match & 6123 & 32.6 \\
2 SIMBAD matches & 1313 & 6.9 \\
$\geq 3$ SIMBAD matches & 670 & 3.6 \\
\hline
\end{tabular}

spurious matches between a SIMBAD and a ROSAT source. This estimate ignores effects resulting from inhomogeneities in the surface density of SIMBAD and BRASS entries. We list in Table 1 some statistics of the crosscorrelation.

Clearly, the large majority of SIMBAD matches are real since only $\sim 1.5 \%$ of the sources with one or more SIMBAD entries are expected to be spurious. Considering the restrictive aim of this work, we did not investigate in detail the reasons explaining the relatively large number of sources with multiple SIMBAD matches. Probably, the high surface density of SIMBAD entries in some specific small regions of the sky, multiple stellar systems and overall the fact that a number of astrophysical objects still appear as distinct SIMBAD objects could account for this effect.

Most of the proposed SIMBAD identifications of BRASS sources $(61 \%)$ are with stars and only $20 \%$ with galaxies and AGN. The high identified stellar fraction obviously reflects the emphasis put on stars in SIMBAD.

The quality of the cross-correlation may be judged from Fig. 1 which shows the relation between Einstein IPC and ROSAT PSPC count rates for the 1244 Einstein sources recovered in the BRASS. IPC rates were extracted from the corresponding measurement headers in SIMBAD. The fractions of ROSAT/Einstein sources with one, two or more than 2 matches in SIMBAD are $77 \%$, $16 \%$ and $7 \%$ respectively. The ratio of the count rates of the two instruments strongly depends on source spectrum with an additional scatter due to variability. We show for comparison two relations, PSPC $=1 \times \mathrm{IPC}$ and $\mathrm{PSPC}=6 \times$ IPC corresponding to hard (power law photon index $=0$ ) and soft (thermal spectra with $\log T=5.8$ ) sources respectively. As expected, most sources lie between these two extreme cases. The outstanding source at IPC = $0.02 \mathrm{cts} \mathrm{s}^{-1}$ and PSPC $=20 \mathrm{cts} \mathrm{s}^{-1}$ is the low-mass X-ray binary transient EXO 0748 - 676 which was detected by Einstein in the low state before its discovery by EXOSAT in outburst.

\subsection{X-ray and optical properties of identified BRASS sources}

For source classification purposes, the most interesting parameters are flux ratios in various energy bands, including the conventional X-ray hardness ratios, but also $F_{\mathrm{X}} / F_{\mathrm{opt}}$

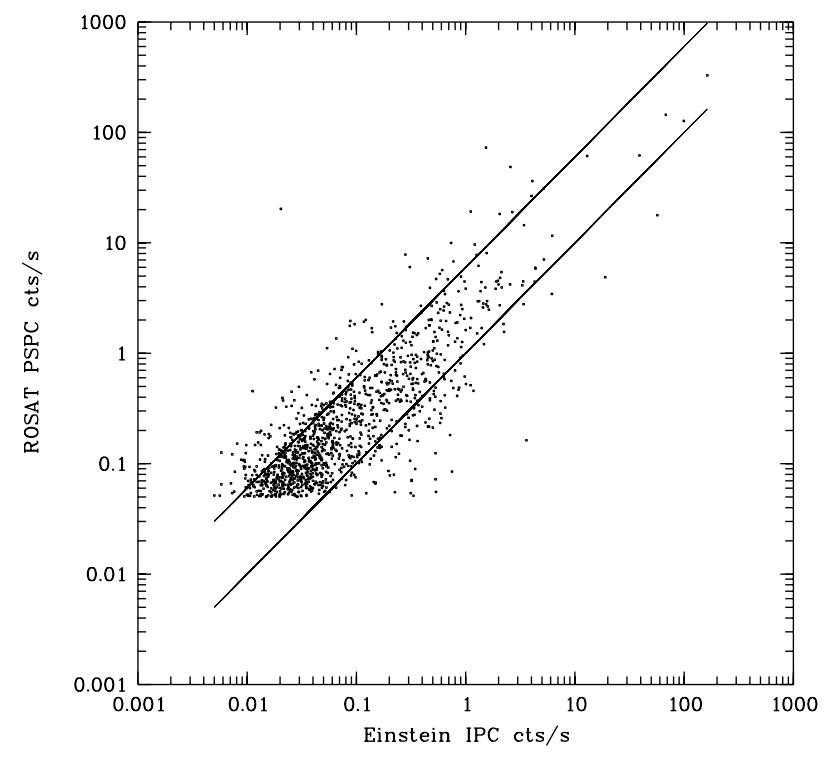

Fig. 1. The relation between Einstein IPC and ROSAT PSPC count rates for 1244 Einstein sources identified with BRASS entries. The two lines represent relations expected for soft and hard sources

ratios as well as optical colours. Another important information for galactic studies is the line of sight absorption which can be estimated from $\mathrm{H}$ I and $\mathrm{CO}$ data.

Arguments involving the $F_{\mathrm{X}} / F_{\text {opt }}$ ratio have been used by many authors in the context of the Einstein Observatory surveys at high and low galactic latitudes (e.g., Maccacaro et al. 1982; Hertz \& Grindlay 1984). ROSAT offers a slightly improved spectral response compared to Einstein and allows PSPC hardness ratios to be used as additional information, at least for relatively bright sources.

\subsubsection{Hardness ratios}

We plot in Fig. 2 the positions in the hardness ratio diagram of BRASS sources cross-identified in SIMBAD with different classes of objects.

The exact energy range used for the computation of hardness ratios changes with the version of the Scientific Analysis System Software (SASS; Voges et al. 1992). Whereas the 93 sources discussed later in this paper were the output of SASS-I (see Sect. 3.1), the BRASS catalogue (Voges et al. 1996) uses SASS-II processing in which the hardness ratios are defined as:

$$
\begin{aligned}
& H R 1=\frac{(0.5-2.0)-(0.1-0.4)}{(0.1-0.4)+(0.5-2.0)}(\mathrm{SASS}-\mathrm{II}) \\
& H R 2=\frac{(1.0-2.0)-(0.5-1.0)}{(1.0-2.0)}(\mathrm{SASS}-\mathrm{II})
\end{aligned}
$$

where $(A-B)$ is the raw background corrected source count rate in the $A-B$ energy range expressed in keV. 
Active coronae populate the central part of the HR1/HR2 diagram with some tail extending towards hard spectra. Stellar coronae are known to exhibit a range of temperatures between $310^{6} \mathrm{~K}$ and $10^{7} \mathrm{~K}$, with the most active and luminous stars also exhibiting the highest $k T$ and hardest spectra. In this diagram, isolated white dwarfs are all found at $H R 1 \leq-0.9$. At the BRASS flux level, the vast majority of active coronae are located within 100 pc from the Sun (e.g. Guillout et al. 1996b) and except for the early type stars and some of the most luminous late type binaries, the effects of interstellar absorption are negligible in both X-ray colours.

In general, the AGN found in SIMBAD do not exhibit $H R 2$ smaller than $\approx-0.2$ but have a large scatter in $H R 1$ which is closely related to galactic foreground absorption (see below). However, ROSAT has discovered some AGN with very steep spectra which are so far barely represented in SIMBAD (e.g. Greiner et al. 1996).

The bulk of the cataclysmic variables populate the upper right quadrant, avoiding very hard and very soft $H R 2$ values. Among outstanding sources we find at $H R 1=$ $0.95 \pm 0.05$ and $H R 2=-0.63 \pm 0.08$ the peculiar soft IP candidate RX J1914.4+2456 which exhibits strong interstellar absorption (Haberl \& Motch 1995; Motch et al. 1996). The supersoft X-ray emission from GQ Mus $=$ Nova Muscae 1983 at $H R 1=-0.05 \pm 0.27$ and $H R 2=-0.87 \pm$ 0.47 was discovered by Ögelman et al. (1993). Cataclysmic variables with $H R 1 \leq 0.1$ are all polar or soft intermediate polar systems.

Most X-ray binaries exhibit very hard X-ray hardness ratios resulting both from the usually high interstellar absorption towards these remote sources and from an intrinsically hard spectrum $(k T \geq 2 \mathrm{keV}$ with sometimes local photoelectric absorption such as in $\mathrm{Be} / \mathrm{X}$ ray binaries for instance). The two objects at the lower left corner are supersoft sources in the Large Magellanic Cloud. The galactic supersoft source RX J0925.7 - 4758 $(H R 1=1.00 \pm 0.01$ and $H R 2=-0.36 \pm 0.08)$ exhibits an intrinsically soft spectrum affected by strong interstellar absorption. The source at $H R 1=0.64 \pm 0.02$ and $H R 2=0.09 \pm 0.02$ is the LMC transient $1 \mathrm{~A} 0538-66$ and that at $H R 1=0.20 \pm 0.03$ and $H R 2=0.31 \pm 0.04$ is the peculiar M giant binary HD 154791/A 1704+241.

\subsubsection{Hardness ratios and $F_{\mathrm{X}} / F_{\mathrm{opt}}$}

The most informative diagrams are without doubt those involving optical information, basically in the form of the $F_{\mathrm{X}} / F_{\mathrm{opt}}$ ratio. The $\mathrm{X}$-ray to optical flux ratio can be defined as $\log \left(F_{\mathrm{X}} / F_{\text {opt }}\right)=\log (\mathrm{PSPC}$ count rate $)+$ $V / 2.5-5.63$, following the expression used by Maccacaro et al. (1982) for the Einstein medium sensitivity survey and assuming an average energy conversion factor of 1 PSPC cts s${ }^{-1}$ for a $10^{-11} \mathrm{erg} \mathrm{cm}^{-2} \mathrm{~s}^{-1}$ flux in the range of 0.1 to $2.4 \mathrm{keV}$. We show in Fig. 3 the position of stars,
AGN, X-ray binaries and cataclysmic variables in the $\mathrm{X}$-ray colour versus $F_{\mathrm{X}} / F_{\text {opt }}$ ratio diagram.

Although stars and AGN have similar X-ray colours, their mean X-ray to optical ratios are obviously quite different and the two populations are well separated in the $H R 1 / 2 F_{\mathrm{X}} / F_{\text {opt }}$ diagram. Stars with $H R 1$ close to -1 are not recorded as white dwarfs in SIMBAD. Their soft X-ray emission could either be due to a particularly low temperature corona or to an unrecognized degenerate companion or nature. They do not appear in Fig. 2 because of their large errors on $H R 2$. X-ray binaries are essentially recognizable from their hard X-ray spectra and usually large $F_{\mathrm{X}} / F_{\mathrm{opt}}$. The low $F_{\mathrm{X}} / F_{\mathrm{opt}} \mathrm{X}$-ray binary tail consists of high mass X-ray binaries. Cataclysmic variables exhibit a large range of X-ray colours and $F_{\mathrm{X}} / F_{\mathrm{opt}}$ ratios and can be somewhat confused with both the AGN and the most active part of the stellar population. However, the addition of a $B-V$ or $U-B$ optical index would allow to distinguish further between these overlapping populations.

\subsubsection{Interstellar absorption}

An efficient way to discriminate between local and remote populations of X-ray sources is to use the anisotropy produced by the Galaxy, essentially in terms of scale height and interstellar absorption. This effect is illustrated in Figs. 4 and 5 which display the position of AGN, CVs and X-ray binaries in the integrated $\mathrm{H} \mathrm{I}$ /hardness ratio diagram. For this study we used $N_{\mathrm{H}}$ data from Dickey \& Lockman (1990).

Most active stars detected in the RASS are little affected by interstellar absorption and their X-ray colours do not vary with galactic latitude nor with integrated column density.

Apparently, the shape of the low energy spectrum of AGN is rather constant with the consequence that hardness ratio 1 is well correlated with the integrated $\mathrm{H}$ I column density as shown in Fig. 4. Actually, this relation is well defined and we used it for preselecting AGN candidates with a high rate of success. However, the $H R 2$ distribution is relatively peaked around a value of 0.1 and does not vary with $N_{\mathrm{H}}$. This is probably due to a selection effect against highly absorbed AGN which are likely to be missing in the mostly optically selected SIMBAD sample.

With X-ray luminosities up to $10^{32-33} \mathrm{erg} \mathrm{s}^{-1}$, cataclysmic binaries can be detected to distances as large as $1 \mathrm{kpc}$ or more at the BRASS sensitivity. There is indeed a slight tendency that cataclysmic variables exhibiting the hardest $H R 1$ are preferentially found at low galactic latitudes but the large variety of spectra emitted in this class (from very soft polars to intrinsically absorbed intermediate polars) somewhat blurs the picture.

In contrast, luminous X-ray binaries are seen by ROSAT at very large distances in deeply absorbed regions of the galactic plane and apart from a few cases 

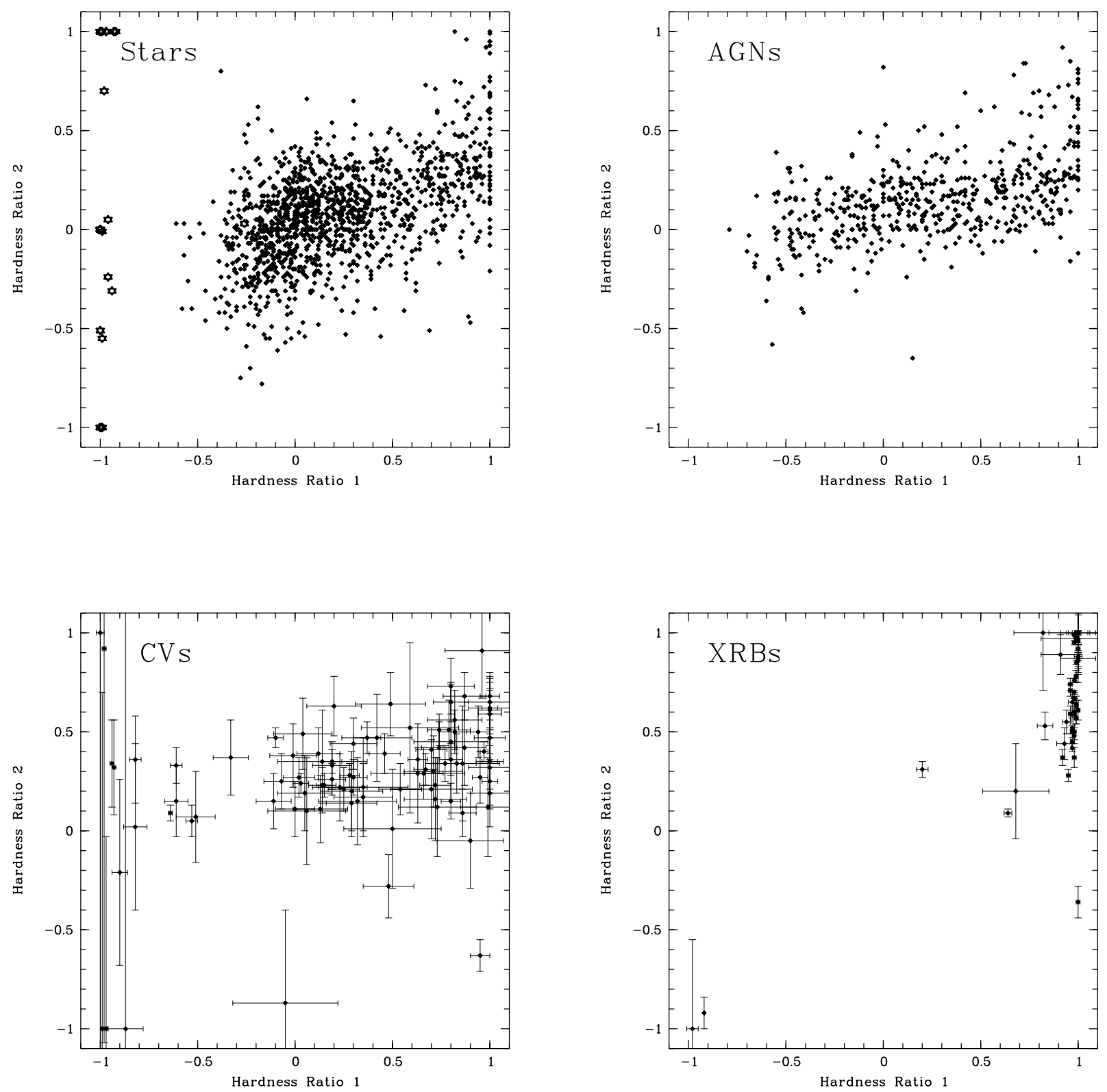

Fig. 2. Position of various classes of X-ray emitters in the $H R 1 / H R 2$ diagram. Asterisks in the Stars diagram represent white dwarfs. The scatter in HR2 of white dwarfs is due to the large error on this ratio for such soft sources. For non-degenerate stars and AGN, we only show sources with errors less than 0.2 on both ratios. The percentages of stars and AGN remaining amount to $40 \%$ and $51 \%$ of the total sample respectively

(e.g. supersoft sources), their hardness ratio $H R 1$ is close to +1 , indicating that all $\mathrm{X}$-ray photons below $0.4 \mathrm{keV}$ are blocked by interstellar absorption. Effects of interstellar absorption are also seen on hardness ratio $H R 2$ which involves higher energy bands. Not surprisingly, Fig. 5 shows that sources lying in the most absorbed direction of the
Galaxy (and therefore lower galactic latitudes) are also the hardest in $H R 2$.

\subsection{Conclusions}

Stars and AGN numerically dominate the ROSAT X-ray sky. These two populations can be easily distinguished 

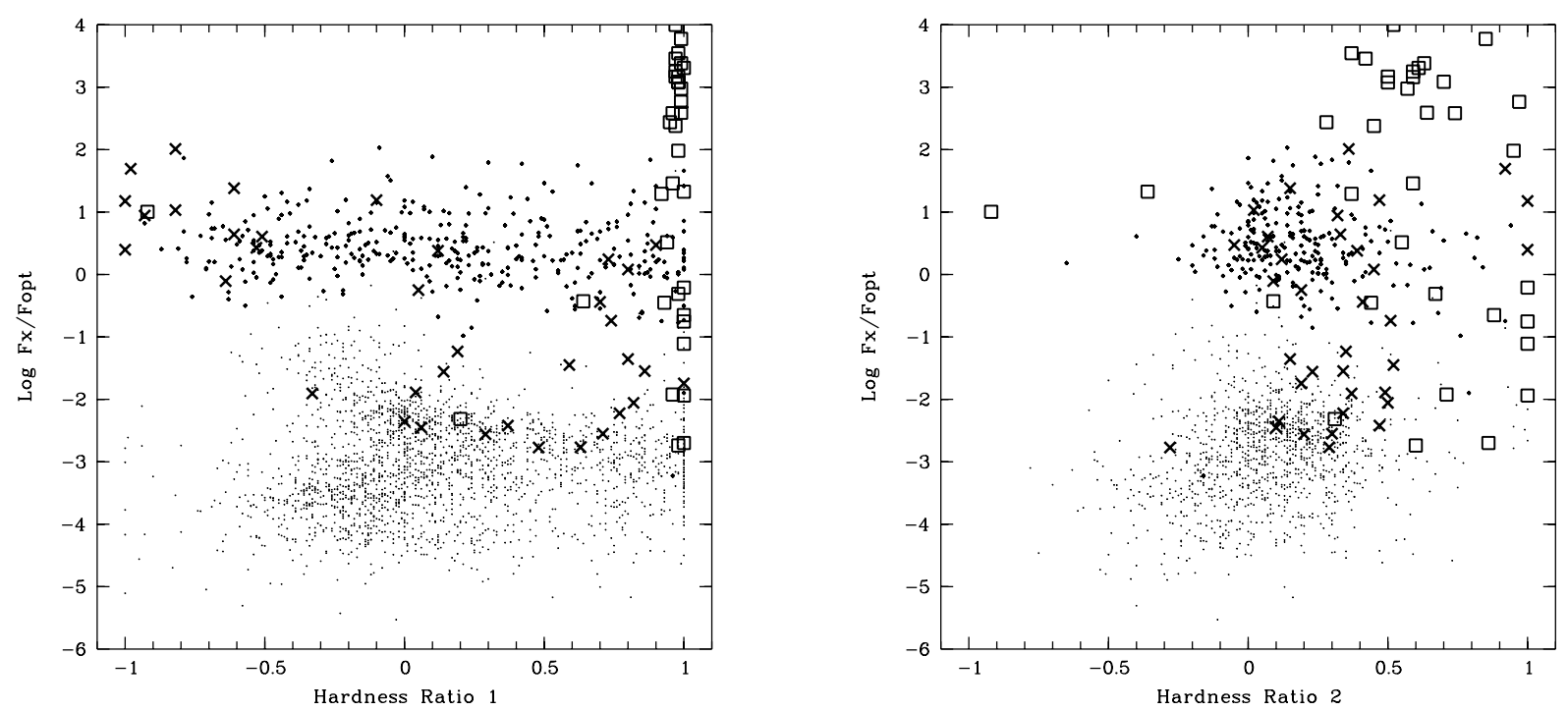

Fig. 3. Position of various classes of identified BRASS sources in the $H R 1$ (left) or $H R 2$ (right) $/ \log \left(F_{\mathrm{X}} / F_{\text {opt }}\right)$ diagram. Small dots represent stars, large dots AGN, squares are X-ray binaries and crosses are cataclysmic variables. For stars and AGN only sources having errors on $H R 1$ or $H R 2$ smaller than 0.2 are plotted
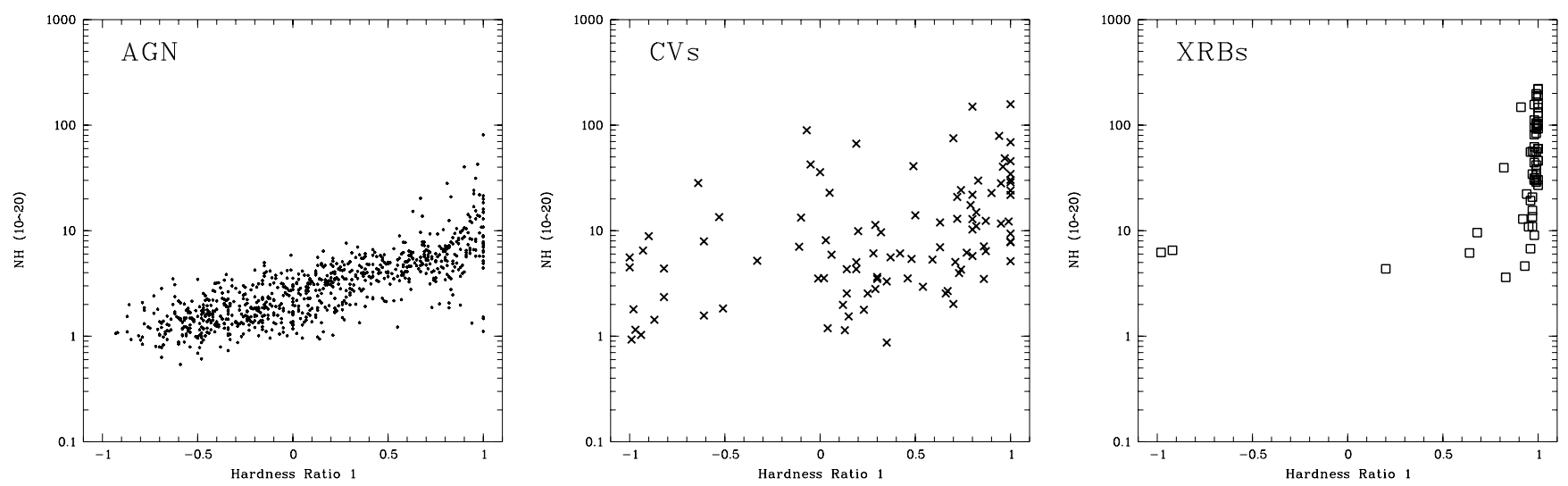

Fig. 4. Variation of $H R 1$ with integrated galactic $\mathrm{H}$ I column density for various classes of X-ray emitters. For AGN identifications, only sources with error on $H R 1$ smaller than 0.2 are plotted

using optical flux information. Searching for rarer X-ray emitters can be made much more efficient by selecting particular domains of the X-ray and optical source parameter space. Some classes of X-ray sources preferentially populate particular regions of the $H R 1 / H R 2$ diagram. For instance, isolated hot white dwarfs, high magnetic field polars and unabsorbed supersoft sources all show very soft spectra. These three classes may be distinguished further from their X-ray to optical flux ratio. X-ray binaries and hard intermediate polar CVs may also be detected from their particularly hard X-ray emission in $H R 1$ and $H R 2$. However, since the hard region also contains some very active coronae and absorbed AGNs, the selection criterion is less efficient than for other classes of sources. The soft $H R 2$ / hard $H R 1$ region has special interest since it is essentially void of stars, AGN, CVs and classical XRBs. This is the place where one expects to find luminous galactic supersoft sources. These general guidelines were used to select the bulk of the sources discussed in the next section. 

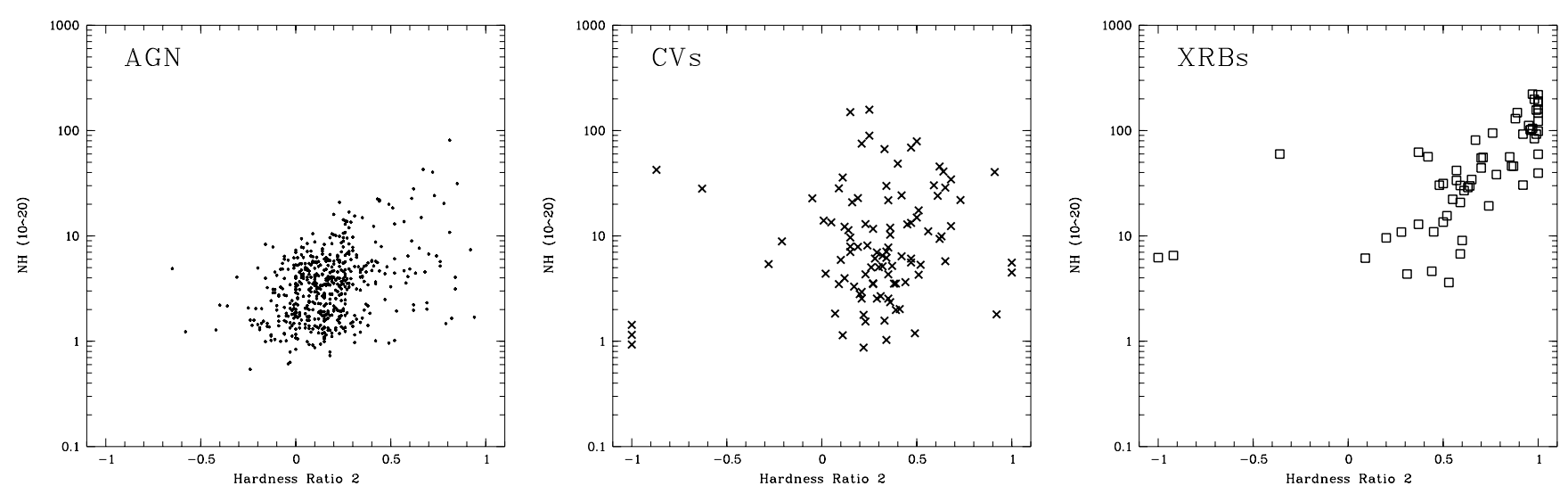

Fig. 5. Variation of $H R 2$ with integrated galactic $\mathrm{H}$ I column density for various classes of X-ray emitters. For AGN identifications, only sources with error on $H R 2$ smaller than 0.2 are plotted

\section{Identification of sources from the ROSAT Galactic Plane Survey}

\subsection{The RGPS source list}

The 93 sources discussed in this paper were extracted from the RGPS source list using selection criteria described in section 3.5 below. The RGPS source list results from the survey reduction performed by SASS-I in October 1991. This RASS reduction has well known documented flaws, namely a PSPC count rate overestimated by a factor of $\approx 1.2$, the appearance of some ghost sources caused by problems in the satellite attitude reconstruction in spinning mode and uneven sensitivity due to the strip accumulation.

Spacecraft problems prevented the all-sky detection of sources between ecliptic longitudes $41^{\circ}$ and $49^{\circ}$ and ecliptic longitudes $221^{\circ}$ and $229^{\circ}$ leaving about $5 \%$ of the galactic sky without coverage.

The 1991 SASS-I version stacked photons in $2^{\circ}$ wide strips along the great scan circles resulting in frequent multiple detections of the same source in adjacent strips (if overlapping sufficiently). The lists of sources derived from each strip were merged into a single master list totaling about 15000 sources at $|b| \leq 20^{\circ}$. This list constitutes the database for the RGPS. Details on the merging procedure can be found in Motch et al. (1997b).

Errors on ROSAT X-ray positions are quadratic sums of the statistical uncertainty with which the centroid of the $\mathrm{X}$-ray image is positioned on the pixel grid by the Maximum Likelihood source detection algorithm and of a systematic attitude error, estimated to be of the order of $8^{\prime \prime}$ (Motch et al. 1997b).

Among the 93 sources, a total of 87 are bright enough to be also found in the RASS bright source catalogue
(1 RXS, Voges et al. 1996) discussed in the previous section. The SASS process used for the production of the bright source list (SASS-II) detects sources on square sky areas, eliminating thus the uneven sensitivity resulting from the strip approach used in 1991. Updated attitude reconstruction removed ghost sources sometimes present in the 1991 version and human data screening ensured high quality. Most RGPS and BRASS positions are fully consistent with a median difference in position of $7.6^{\prime \prime}$. The $90 \%$ confidence radii are similar. In a few cases mentioned below in the notes on individual objects, the offset between the RGPS and BRASS positions is significantly larger than $r_{90}$ probably because of the use of an improved attitude solution in the SASS-II reduction. The 1991 SASS-I reduction also uses slightly different energy ranges than the SASS-II reduction for computing hardness ratios 1 and 2 :

$$
\begin{aligned}
& H R 1=\frac{(0.40-2.40)-(0.07-0.40)}{(0.07-2.40)}(\mathrm{SASS}-\mathrm{I}) \\
& H R 2=\frac{(1.00-2.40)-(0.40-1.00)}{(0.40-2.40)}(\mathrm{SASS}-\mathrm{I})
\end{aligned}
$$

where $(A-B)$ is the raw background corrected source count rate in the $A-B$ energy range expressed in keV. There is no one to one relation between SASS-I and SASSII hardness ratios as their values depend on the details of the observed count distribution in energy. However, a meaningful average graphical relation can be estimated using $H R 1$ and $H R 2$ plots for sources with accurate hardness ratio determinations. This relation was used to propagate the ranges from one reduction to the other.

\subsection{Optical observations}

Optical material was collected at the Observatoire de Haute-Provence, CNRS, France for northern sources and 
at ESO, La Silla for the southern sky. The present observational material was acquired during several runs dedicated to the identification of ROSAT galactic plane survey sources in general which were carried out from 1991 till 1995 by various observers.

At OHP, multicolour CCD imagery was usually obtained with the $1.2 \mathrm{~m}$ telescope few days before the spectroscopic run with the $1.9 \mathrm{~m}$. In most cases we used $B$ and $I$ band filters with additional $U$ band exposures in a few instances. Pixel size was 0.85 or 0.77 arcsec on the sky depending on the CCD chip used. At the $1.9 \mathrm{~m}$ telescope, we operated the CARELEC spectrograph (Lemaitre et al. 1990). Most of the time we used two dispersions, a low resolution mode $260 \AA / \mathrm{mm}(\lambda \lambda 3500-7500 \AA$; FWHM resolution $\approx 14 \AA$ ) and a medium resolution mode in the blue $33 \AA / \mathrm{mm}(\lambda \lambda 3800-4300 \AA$; FWHM resolution $\approx 1.8 \AA$ ).

ESO data were acquired at the occasion of four runs in May 1991, April 1992, February 1994 and February 1997. In 1991, 1994 and 1997, we used the Boller \& Chivens spectrograph at the ESO $1.5 \mathrm{~m}$ telescope. Medium dispersion gratings were used in all cases yielding a FWHM resolution of $4-5 \AA$ and a wavelength range $\lambda \lambda 3900-7200 \AA$. In 1992 we used EFOSC 2 at the ESO-MPI 2.2 m telescope with the same instrumental setting as described in Motch et al. (1994).

All spectral and photometric data reductions were performed using standard MIDAS procedures (Banse et al. 1983). Spectra were corrected for bias and flat-field and later calibrated in wavelength using arc lamps. In most cases we could acquire flux standard stars. However, uncertain weather conditions and the narrow slit entrance width sometimes used may introduce large errors in the derived flux. These errors may be of the order of a factor 2 or more.

\subsection{Optical data analysis}

For active coronae, spectral classification was carried out as outlined in Motch et al. (1997a) using Turnsheck et al. (1985), Jacoby et al. (1984) and Jaschek \& Jaschek (1987) stellar atlases.

Visual magnitudes were in most cases not derived from our CCD imagery as they usually lacked photometric calibration. Instead we used magnitudes extracted from the SIMBAD database, the Guide Star Catalogue (Lasker et al. 1990) or for the faintest counterparts, from the USNO-A1 catalogue (Monet 1997). The GSC magnitudes of stars having a spectral type were corrected for colour effects according to relation (1) of Russell et al. (1990) assuming a main sequence unreddened object. After colour correction, the remaining $1 \sigma$ error on magnitudes is $\approx 0.2$ mag.

The coordinates of the optical counterparts were in first priority extracted from the SIMBAD database which usually gives entries from astrometric catalogues (e.g. PPM). When no accurate SIMBAD positions were available we used the GSC coordinates and for the remaining identifications positions computed interactively using the Aladin sky atlas (Bonnarel et al. 1997) at the Centre de Données de Strasbourg (CDS).

The Aladin project aims to provide multi wavelength cross-identification. This tool is designed as an interactive $\mathrm{X}$-window client accessing images from the CDS image server, Simbad database, CDS catalogue server and onsite catalogues. The Aladin collection contains a high resolution image archive of Schmidt plates digitized by the Paris MAMA facility and covering a significant portion of the sky, mainly in the Magellanic Clouds and southern Galactic Plane. The integration of the STScI Digital Sky Survey -1 in the system provides full-sky coverage albeit with a lower spatial resolution and astrometric accuracy than that of the MAMA archive. For the plates digitized by the MAMA, astrometric calibration is based on PPM standards and reaches an accuracy better than $0.3^{\prime \prime} \mathrm{rms}$.

\subsection{Optical identifications}

The strategy used for optical identification of ROSAT sources in the galactic plane has been extensively discussed in Motch et al. (1997a). For stars, we used two criteria based on the Ca II H\&K or $\mathrm{H} \alpha$ flux to X-ray flux ratio and a priori probability of positional coincidence in the relatively small X-ray $r_{90}\left(<r_{90}>=25^{\prime \prime}\right)$. A star was identified as the counterpart of the X-ray source when its line emission level was compatible with the X-ray flux, or when no spectral line could be measured, on the basis of positional coincidence. The surface density of optically bright active galactic nuclei, cataclysmic variables, hot white dwarfs and Be stars is small enough that the discovery of one such object in the ROSAT error circle is highly significant.

For all identified sources but one (RX J0254.6+3931) we show an optical spectrum of the counterpart, either low or medium resolution but in general, do not provide finding charts as the identifier and positions are in principle sufficient to localize the object. However in few cases where the counterpart does not appear in the USNO catalogue we show finding charts. We also show finding charts for all accreting sources.

For the 17 cases where we failed to find the counterpart we show on a finding chart the observed candidates in order to ease further follow-up studies but do not plot the spectra.

By default, the finding charts are extracted from the STScI DSS- 1 and the RGPS/SASS-I 90\% confidence error circle is shown. In some instances where the DSS-1 data are not able to show the candidates because of crowding or extreme colours, we use instead our CCD images.

Comments on individual sources, spectra and finding charts are given in Sect. 5 . 


\subsection{Source selection}

The ROSAT sources discussed in this paper were extracted from the entire RGPS source list covering the whole galactic plane (apart from the small interruptions mentioned above). We mainly used four different selection criteria, hard, soft, absorbed soft and bright candidates, all based on SASS-I hardness ratios and count rates. Hardness ratio boundaries were chosen following the observed repartition of identified sources in the preliminary cross-correlation made in 1991 (Motch 1991a). These installments of X-ray selected sources were then distributed among several optical groups for identification at the telescope. In this paper, we present the optical work done by one of these observing groups. Some optical identifications which have been already published in dedicated papers are not repeated here whereas work at the telescope is pursued for a number of other sources.

To these purely X-ray selected samples, we add a couple of AGN extracted from not yet fully published lists of identifications in sample areas (e.g., GPS1-4; Motch et al. 1991b, Taurus region; Guillout et al. 1996a). Three other sources are from a so far barely investigated region located at $l \sim 130^{\circ}$. In the following, we consider all sources together independently of their original selection. A small fraction of these sources was re-observed by the HRI in order to get more accurate positions. These cases are mentioned in Sect. 5.

Hard sources were defined as having $H R 1 \geq 0.7$ and $H R 2 \geq-0.1$ (HR1 $1_{\mathrm{BRASS}} \geq 0.65$ and $\left.H R 2_{\mathrm{BRASS}} \geq-0.15\right)$. We furthermore imposed the additional condition that integrated galactic absorption had to be larger than $410^{21}$ $\mathrm{H}$ atom $\mathrm{cm}^{-2}$ and rejected extended sources. The requirement of large galactic absorption was aimed at screening the extragalactic component and searching preferentially for galactic accreting binaries. Applied to the entire RGPS database, this selection yielded 157 entries with count rates larger than 0.1 cts s $^{-1}$ among which 84 had no identification in SIMBAD. We report here on 26 of these unidentified sources. We added to this sample an additional set of 13 sources sharing constraints on $H R \mathrm{~s}$ but not on $N_{\mathrm{H}}$ and flux. These sources arise from early selections or from sample areas.

Soft sources were defined as having $H R 1 \leq-0.4$ $\left(H R 1_{\mathrm{BRASS}} \leq-0.75\right)$ with a maximal error of $0 . \overline{5}$ and a PSPC count rate $\geq 0.1 \mathrm{cts} \mathrm{s}^{-1}$. Such soft sources do not emit much at energies higher than $0.4 \mathrm{keV}$ and $H R 2$ is therefore essentially undefined. A total of 112 sources fulfilled this criterion among which 43 had no identification in SIMBAD. We expected to preferentially find white dwarfs and in general low luminosity soft sources in this sample. Several of these sources were also detected by the Wide Field Camera and subsequently identified.

Absorbed soft sources were defined as having $H R 1 \geq-0.4$ and $H R 2 \leq-0.4\left(H R 1_{\mathrm{BRASS}} \geq-0.6\right.$ and $\left.H R 2_{\mathrm{BRASS}} \leq-0.2\right)$. This sample was designed to discover
Table 2. Repartition of the number of sources by selection criteria

\begin{tabular}{lc}
\hline $\begin{array}{l}\text { Selection } \\
\text { Criteria }\end{array}$ & $\begin{array}{c}\text { Number } \\
\text { of sources }\end{array}$ \\
\hline Hard & 39 \\
Soft & 9 \\
Absorbed soft & 19 \\
Bright & 17 \\
Other & 9 \\
\hline
\end{tabular}

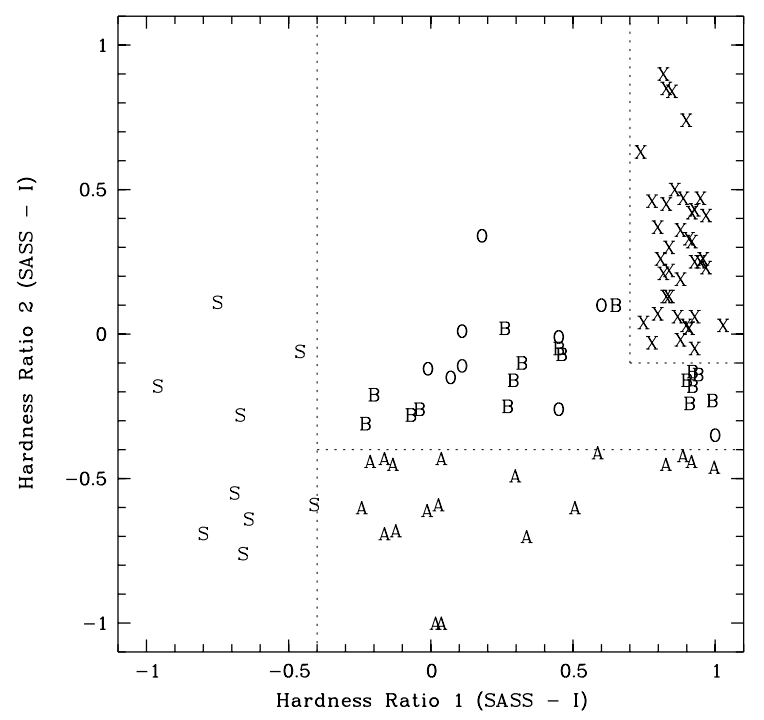

Fig. 6. Position of the various X-ray selected samples in the $H R 1 / H R 2$ diagram. $\mathrm{X}=$ hard $\mathrm{X}$-ray binary like, $\mathrm{A}=$ absorbed supersoft candidates, $\mathrm{S}=$ soft white dwarf candidates, $\mathrm{B}=\mathrm{X}$ ray bright sources, $\mathrm{O}=$ other remaining sources

intrinsically soft luminous sources undergoing relatively large interstellar absorption as a result of their remote location. Above $0.1 \mathrm{cts} \mathrm{s}^{-1}, 667$ RGPS sources fulfil these constraints among which 199 had no identification in SIMBAD.

Sources which do not fall in any of the other hardness ratio ranges are split into the "Bright" group (PSPC count rates larger than $0.25 \mathrm{cts} \mathrm{s}^{-1}$ ) and the "Other" group.

All samples were cleaned by discarding those sources having an obvious identification in SIMBAD (catalogued $\mathrm{X}$-ray source, bright active corona). However, in the course of this identification programme, some of these sources were recovered by other instrumentations (Sky Lab SLX, WFC RE, EUVE, etc.) or identified by other groups and now appear as such in SIMBAD.

We show in Fig. 6 the position of the various X-ray selected samples in the $H R 1 / H R 2$ diagram and give number repartition by selection criteria in Table 2 . 
In Table 4 we list the RGPS source positions, count rates, hardness ratios and 1 RXS identification when available. Table 5 gives optical identification, position, $V$ and $B$ when available. Spectra are shown in Fig. 8 and finding charts in Figs. 9 and 10.

\subsubsection{Hard sources}

Among the already published identifications of hard sources are the four CVs RX J0028.8+5917, RX J0744.9-5257, RXJ1141.3-6410 and RX J2123.7+ 4217 (Motch et al. 1996). Some of the Be/X-ray candidates reported in Motch et al. (1997b) or the low-mass X-ray binary GS1826-24 identified in Barret et al. (1995) were also found in this sample. Here we report on the identification of RX J1739.5-2942 with a new Be/X-ray binary (see below). Our hard sample also contains the ultrasoft transient SLX 1746-331 (Skinner et al. 1990) which was apparently in outburst at the time of the ROSAT survey observation in September 1990 and not detected in follow-up ROSAT HRI observations on 1994 October 2.

Because of observational constraints, SIMBAD contains hardly any AGN seen through large galactic foreground absorption whereas all luminous galactic X-ray binaries, mostly discovered at higher energies are listed. This strong bias indicates that in hard X-ray selected samples, the number ratio of AGN to X-ray binaries or CVs should be much larger than suggested by the BRASS-SIMBAD correlation (see Figs. 2, 4 and 5). A total of 14 AGN are indeed found in the hard sample (see Table 3), confirming that in the galactic plane, there is no easy way to disentangle absorbed AGN from genuine accreting binaries since both populations exhibit hard spectra and faint bluish counterparts. Not surprisingly, the new identified hard AGN are much more absorbed than the SIMBAD sample and their $H R 2$ is correlated with galactic $N_{\mathrm{H}}$ (see Fig. 7). In 10 instances we identify the X-ray source with an optically bright active corona and in 13 cases, we fail to find a likely counterpart. Not unexpectedly, the vast majority of unidentified sources (13 among 17) are found in the hard sample and based on the properties of the identified population we can predict that most of these sources are likely to be absorbed extragalactic objects. In particular, from the lack of bright objects in $I$ band images we can exclude relatively close $\mathrm{Be} / \mathrm{X}$-ray binaries as possible identification for these hard sources.

\subsubsection{Soft sources}

The majority of the very soft sources discovered in the RASS turned out to be white dwarfs often also detected as bright UV sources in the Wide Field Camera Survey (Pounds et al. 1993) or by the Extreme Ultraviolet

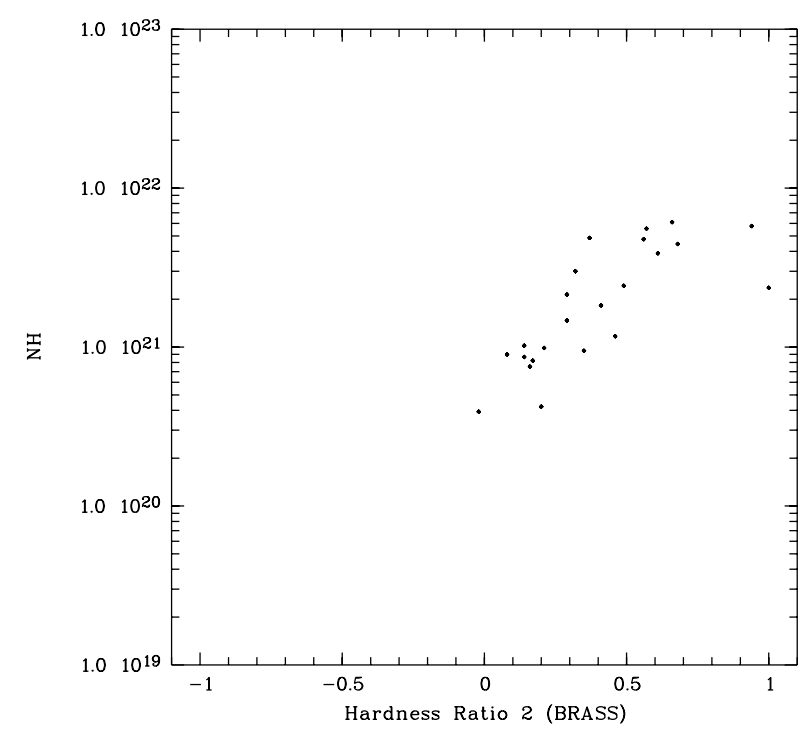

Fig. 7. Relation between hardness ratio $H R 2$ and galactic absorption for the newly identified AGN

Explorer (Malina et al. 1994). Many of these bright sources were readily optically identified from their UV positions (Mason et al. 1995). Again, some active coronae emit low temperature X-ray spectra which are indistinguishable from white dwarf emission at the PSPC survey sensitivity.

Among the already published discoveries in this soft sample are the extremely hot PG 1159 star RX J2117.1+3412 (Motch et al. 1993) and the hot WD RX J2052.7+4639 (Motch et al. 1997a).

The other class appearing in this X-ray selected group is that of soft polars and intermediate polars in which the blackbody like X-ray emission from the polar cap dominates over the hard bremsstrahlung radiation from the shock in the accretion column (Beuermann \& Schwope 1994; Haberl \& Motch 1995). The polar RX J1802.1+1804 (Greiner et al. 1995) and RE 0751+14 (Mason et al. 1992; Motch \& Haberl 1995) were also found in this sample. The exotic population of isolated neutron stars accreting from the interstellar medium should also show up as soft sources. In fact, two of the best candidates known so far were found in the soft sample (RXJ1856-37 Walter et al. 1996, RX J0720-3125 Haberl et al. 1997). RX J2102.0+3359 (see Tables 4 and 5) was also considered for some time as a good lone neutron star candidate. However, UB CCD imagery revealed the presence of a faint UV excess object (star $\mathrm{X}, B=21.6, U-B=-1.1$ ) close to the center of the error circle. This picture hints towards identification with an extreme $F_{\mathrm{X}} / F_{\text {opt }}$ ratio AM Her CV such as RXJ0453.4-4213 = RS Cae (Burwitz et al. 1996). However, its firm identification awaits optical spectroscopic confirmation. 
These identifications are in agreement with the results of the BRASS-SIMBAD analysis discussed above.

In the soft sample presented here, we report the identification of a new DA white dwarf (RX J1936.3+2632 $=2 \mathrm{EUVE}$ J1936+26.5), 2 new polars (RX J0649.8-0737 and RX J0749.1-0549) of rather long orbital period and of a couple of active coronae.

\subsubsection{Absorbed soft sources}

Based on the obvious observation that no extremely X-ray bright soft source was found in the ROSAT survey nor in previous EUV surveys, we concluded that if a luminous supersoft source analogue to Cal 83 in the LMC were existing in the Galaxy, its observed X-ray spectrum would be dimmed and distorted by interstellar absorption. In order to delimit the range of hardness values to select, we simulated soft black body spectra affected by large interstellar absorption. With the ROSAT PSPC, large photoelectric absorption of a very soft source produces a very peaked distribution centered at an energy which depends on the balance between $k T$ and $N_{\mathrm{H}}$. These spectra are characterized by a hard $H R 1$ and a soft $H R 2$.

Unfortunately, at the PSPC resolution, optically thin thermal spectra with many emission lines in the range of $0.3-0.5 \mathrm{keV}$ can also produce peaked spectra, albeit with a much lower photoelectric absorption. Some active coronae may emit this kind of spectra in a narrow range of temperature and photoelectric absorption. Such spectra are also often seen in supernova remnants and in the hot diffuse galactic emission in general. Care was therefore taken to exclude extended X-ray sources by examining visually the survey images of all absorbed soft detections.

The first sample of absorbed soft sources in which we discovered the galactic supersoft binary RX J0925.7-4758 (Motch et al. 1994) and the peculiar intermediate polar candidate RX J1914.4+2456 (Motch et al. 1996) had more stringent constraints than set here $\left(H R 1_{\text {SASS-I }} \geq-0.4\right.$ and $H R 2_{\text {SASS-I }} \leq-0.6$ with errors on $H R 1$ and $H R 2$ of less than 0.5 and 1.0 respectively and a count rate larger than $0.1 \mathrm{cts} \mathrm{s}^{-1}$ ). This restricted sample is now mostly identified and the remaining sources are associated with active stars.

Relaxing the constraints on $H R 2$ increased the number of candidates but also the fraction of active coronae. Therefore, apart from one AGN (RX J1929.8+4622) and two CVs (RX J1951.7+3716 and RX J1946.2-0444), most sources are identified with stars. In three cases, however, we fail to identify the source. A fraction of these unidentified sources could be AGN with absorbed steep spectra.

\subsubsection{Bright sources}

A number of unidentified bright RGPS sources of high $F_{\mathrm{X}} / F_{\text {opt }}$ turned out to be previously unknown AGN or
CVs. As shown in Fig. 3, selecting high $F_{\mathrm{X}} / F_{\text {opt }}$ sources at intermediate hardness ratios does improve the fraction of extragalactic and cataclysmic variable identifications, however, with slightly less efficiency than other HR based selections. Some very active coronae with magnitudes below that of the limit of HD or SAO catalogues were also discovered.

\section{Conclusions}

The BRASS-SIMBAD cross-correlation and the set of optical identifications presented in this paper show that careful selection on multi-wavelength parameters can help to disentangle the various populations of X-ray sources accounting for the ROSAT survey.

In order to reach the state at which one can really compute probabilities of identification of any source with the main classes of X-ray emitters, large source samples have to be completely identified using catalogue data and proper multi-wavelength observations. In the galactic plane, the level of screening of the extragalactic population by absorption and in general galactic latitude are key parameters. Ideally, selected test samples should be accumulated at various latitudes in order to properly handle likelihoods of identification such as for instance outlined in Sterzik et al. (1995).

Among the 93 selected sources, we find a likely counterpart to the X-ray source in 76 cases. With 39 identifications, active coronae again dominate source count. However, X-ray selection enhances the number of noncoronal identifications and we report on 25 new AGN, 6 new CVs, 2 new white dwarfs and a new Be/X-ray binary.

Our programme of optical identification of X-ray selected RGPS sources is going on and new identifications will be reported in a forthcoming paper. Statistics of identifications in the various selected samples will also be presented at this occasion.

\section{Notes on individual objects}

\subsection{Stars}

RX J0222.5+5033 = BD+49 646: $\mathrm{BD}+49646$ was also detected by the ROSAT WFC (2RE J0222+503, Pye et al. 1995). The star GSC0330200570 located NE from BD+49 646 is a late A type star unrelated to the X-ray source.

RX J0621.2+4415 = G 101-35: The BRASS error circle now encompasses the Me star. The star has high proper motion.

RX J0635.9+0755 = GSC0073302098: The candidate star GSC0073302098 lacks the strong CaII H\&K emission which would be expected based on the PSPC count rate. However, the a priori probability of coincidence of the ROSAT source with the late $\mathrm{G}-\mathrm{K} V \approx 10.7$ star is small 
enough $\left(510^{-3}\right)$ so that the identification can be considered. The ROSAT source is identified with $2 \mathrm{E} 0633.2+075$.

RX J0702.0+1257 = GSC0075701608: Using EUVE data, Vennes et al. (1997) identify the source as a K0IV-V and a hot white dwarf in a wide binary. Part of the softness of the PSPC spectrum could be indeed explained by a contribution from the degenerate star. The XRT source is identical with EUVE J0702+129 and 2RE J0702+125 (Pye et al. 1995).

RX J0704.5-0612 = GSC0482603053 ??: The brightest object in the RGPS error circle is GSC0482603053. A medium resolution spectrum of the star reveals CaII $\mathrm{H} \& \mathrm{~K}$ emission and some evidence for weak $\mathrm{H} \alpha$ emission. Unfortunately, no absolute CaII flux could be measured for this active corona, leaving some doubt on the compatibility of the chromospheric signature with the ROSAT X-ray flux. The second brightest object located SW from GSC0482603053 is a late type star without marked activity.

RX J0713.1-0511 = GSC0482302265: The XRT source is identical to 2RE J0713-051.

RX J0721.3-5720 = GSC0855901016 + comp: The candidate M5e star exhibits strong Balmer emission and is probably responsible for a large part of the $\mathrm{X}$-ray emission. The close and comparatively bright star $(V \approx 11)$ GSC0855901016 is a main sequence K0 star. Unfortunately, we do not have CaII H\&K spectra which would have given indication on the level of stellar activity. The a priori coincidence of position between GSC0855901016 and a ROSAT survey source is small (8.2 $10^{-3}$ ) and could indicate that the bright star also contributes to the X-ray emission and constitutes a physical pair with the Me star.

RX J0811.1-5555 = GSC0857001980: The absence of a spectrum covering the CaII H\& $\mathrm{K}$ region does not allow to firmly identify this source. However, in this case, the BRASS position which has a better accuracy than the RGPS determination, is centered on GSC0857001980 and excludes any other object than the $V \approx 12$ late $\mathrm{G}$ star. This positional evidence (a priori probability of coincidence of $1.310^{-2}$ ) and the soft X-ray hardness ratios suggest that GSC0857001980 is the counterpart of the ROSAT source.

RX J0828.5-5138 = GSC0816200330: The BRASS position is now centered on the G star GSC0816200330. The low $\left(610^{-3}\right)$ a priori probability of a random positional coincidence of the X-ray source with the relatively bright $(V \approx 11)$ star and the soft $\mathrm{X}$-ray hardness ratio suggest a coronal identification.

RX J0845.7-3544 = GSC0714900583: GSC0714900583 is a late $\mathrm{G}$ star, which considering the coincidence in position with the X-ray source $\left(8.310^{-3}\right)$ and the soft X-ray spectrum is most probably the optical counterpart of the $\mathrm{X}$-ray source.

RX J0856.4-2241 = GSC0658500334: Weak Balmer emission may be present in the spectrum of GSC0658500334.
Table 3. Magnitude, redshift and selection of newly identified AGN

\begin{tabular}{cclcc} 
Source Name & $V / B$ & Type & $z$ & Sel \\
\hline RX J0222.1+5221 & 17.00 & Sy 1 & 0.200 & X \\
RX J0254.6+3931 & 16.10 & BLRG & 0.289 & O \\
RX J0324.7+3410 & 15.10 & Sy 1 & 0.063 & X \\
RX J0325.2+4042 & 15.20 & Sy 2: & 0.048 & O \\
RX J0337.0+4738 & 17.00 & Sy 1 & 0.184 & X \\
RX J0434.7+4014 & 15.20 & Sy 1 & 0.021 & X \\
RX J0452.0+4932 & 17.10 & Sy 1 & 0.029 & X \\
RX J0459.8+1808 & 19.50 & Sy 1 & 0.157 & O \\
RX J0508.3+1721 & 13.47 & Sy 2: & 0.017 & X \\
RX J0508.9+2113 & 17.70 & Sy 1 & 0.190 & X \\
RX J0602.1+2828 & 15.00 & Sy 1 & 0.033 & X \\
RX J0608.0+3058 & 17.30 & Sy 1 & 0.073 & X \\
RX J0750.9+0320 & 15.20 & Sy 1 & 0.099 & B \\
RX J0755.7-0157 & 14.80 & Sy 1 & 0.040 & B \\
RX J0801.9-4946 & 11.07 & Sy 1 & 0.040 & X \\
RX J0816.4-1311 & 17.40 & BL LAC & $*$ & B \\
RX J0818.9-2252 & 15.50 & Sy 1 & 0.035 & X \\
RX J0913.0-2103 & 17.30 & BL LAC & $*$ & B \\
RX J1023.9-4336 & 17.50 & BL LAC & $*$ & B \\
RX J1741.4+0348 & 15.30 & Sy 1 & 0.030 & X \\
RX J1929.8+4622 & 17.00 & Sy 1 & 0.127 & A \\
RX J1931.1+0937 & 19.00 & BL LAC & $*$ & X \\
RX J2040.3+1059 & 16.10 & Sy 1 & 0.085 & B \\
RX J2043.9+5314 & 17.50 & Sy 1 & 0.080 & X \\
RX J2044.0+2833 & 14.70 & Sy 1 & 0.050 & B \\
\hline
\end{tabular}

The BRASS position is also centered on the early M star with a similar error radius. The soft X-ray spectrum is also consistent with a coronal identification.

RX J1704.3-4020 = HD 322763: A follow-up $H R I$ observation gives an improved position, $\mathrm{RA}=1704$ 17.68, $\mathrm{DEC}=-401947.6$ centered on the A star. X-ray emission may originate from an undetected late type companion star.

RX J2010.5+0632 = GSC0050701588: The Me star is probably identified with the Parkes-MIT-NRAO survey source PMN J2010+0632 (Griffith et al. 1995). An X-ray flare was detected during the survey.

RX J2014.8+4501 = HD 192785: The K0V star $\mathrm{HD}$ 192785 exhibits $\mathrm{H} \alpha$ re-emission (not shown) and was also detected in the Einstein Slew Survey (Schachter et al. 1996).

\subsection{Active galactic nuclei}

AGN identified in the low galactic latitude regions have all low redshifts and the dominant population is Seyfert 1.

RX J0222.1+5221 = PMM1350-02324516: This identification was already reported in Motch et al. (1991b).

RX J0254.6+3931 = CJ2 0251+393: We did not obtain spectroscopic observation of this quasar. Its identification 
is reported in Vigotti et al. (1989).

RX J0324.7+3410 = GSC0234901904: This Seyfert 1 nucleus was independently discovered and identified with the HEAO-1 source H0321+340 by Remillard et al. (1993) and with the ROSAT source by Kock et al. (1996).

RX J0452.0+4932 = H0432052: This object was already listed by Hauschildt (1987).

RX J1931.1+0937 = A: Follow up ROSAT HRI observations revealed flare like activity by a factor of $\approx 2$ on a time scale of one day. The best HRI position is $\mathrm{RA}=193109.23, \mathrm{DEC}=+093718.96$ with a conservative error radius of $10^{\prime \prime}$. The proposed optical counterpart displays a blue featureless continuum and is located within the $1^{\prime \prime}$ radius error circle of the radio source NVSS J193109+093717. Based on these evidences we propose an identification with a BL Lac type of AGN.

RX J2040.3+1059 = PMM0975-19711620: The AGN could be in an interacting system

\subsection{Accreting sources}

Detailed X-ray and, optical photometric and spectroscopic studies of the new CVs will be presented in future papers.

RX J0649.8-0737 = PMM0750-02887019: This CV is a polar with $\mathrm{a} \approx 4.4 \mathrm{~h}$ orbital period.

RX J0749.1 $-0549=$ PMM0825 -05603282 : $T$ his $C V$ is a polar with a $\approx 3.6 \mathrm{~h}$ orbital period.

RX J1739.4-2942 = A: RX J1739.5-2942 is probably identical with GRS 1736-297 since the ROSAT position is located well within the $90^{\prime \prime}$ radius of the $90 \%$ confidence circle of GRS 1736-197. The Be/X-ray nature of the source is consistent with the hard ART-P X-ray spectrum observed by GRANAT (Pavlinsky et al. 1994).

\subsection{Miscellaneous and optically unidentified}

RX J0035.8+5950 = ??: None of the two GSC stars GSC0366601407 (A) and GSC0366600907 (B) lying at the edge of the ROSAT error circle exhibits detectable chromospheric activity.

RX J0529.0+0934 = ??: Objects A and B display featureless continuum. B is bluer than $\mathrm{A}$.

RX J0602.2+2837 = ??: Object A has a featureless continuum. Object B could exhibit $\mathrm{H} \alpha$ emission.

RX J0620.6+2644 = ??: The BRASS 90\% confidence error area (small circle) does not overlap with the RGPS $90 \%$ error area. Because of the improved reduction process, the BRASS position should be considered as the best one. All objects A-E are late type stars without Balmer emission. A: late K, B: G-K, C: K, D: G-K and E: late G.

RX J0621.7+1747 = ??: Object $A$ is a late $B$ type star, $B$ a $\mathrm{K}$ type star and $\mathrm{C}$ a G-K type star.
RX J0648.7+1516 = ??: The ROSAT source is identical to 1H $0646+152$. A short exposure time spectrum of object A reveals a rather blue featureless continuum. B and C are probably late type stars. The finding chart shows the BRASS position as no reliable error radius was returned by the SASS-I process for this source.

RX J0717.4-1119 = ??: The source was not recovered in a subsequent $H R I$ pointing. Object $\mathrm{A}$ is a $\mathrm{K}$ type star and B has an A spectral type.

RX J0759.1+0748 = ??: Bright X-ray source. Object $A$ has a F-G spectral type and star $\mathrm{C}$ displays $\mathrm{H} \alpha$ and $\mathrm{H} \beta$ in absorption. Objects $\mathrm{D}$ and $\mathrm{C}$ are probably extended and do not exhibit strong emission lines up to $6800 \AA$.

RX J0819.2-0756 = ??: Objects A and B are F-G type stars. Object $\mathrm{C}$ is the only object visible inside the $H R I$ error circle (not shown).

RX J1718.4-4029 = ??: The image shown here is a 5 min $I$ band CCD exposure obtained in April 1992 with EFOSC2 on ESO-MPI $2.2 \mathrm{~m}$. The HRI error circle $(\mathrm{RA}=$ $171824.13, \mathrm{DEC}=-402930.4,90 \%$ confidence radius $\left.=15.7^{\prime \prime}\right)$ is plotted. Objects $\mathrm{A}, \mathrm{B}$ and $\mathrm{C}$ display $\mathrm{H} \alpha$ and $\mathrm{H} \beta$ absorption lines.

RX J1740.7-2818 = ??: The CCD image shown here is a 5 min $I$ band exposure obtained with EFOSC2 and the MPI-ESO $2.2 \mathrm{~m}$ telescope in April 1992. The field is heavily reddened. Objects A, B, C and D are late M stars without any noticeable Balmer emission.

RX J1742.3-2737 = ??: The CCD image shown here is a 2 min $I$ band exposure obtained with EFOSC2 and the MPI-ESO $2.2 \mathrm{~m}$ telescope in April 1992. The field is heavily reddened. Object $\mathrm{A}$ is a reddened A-F type star and object B a reddened G-K star.

RX J1749.8-3312 = SLX 1746-331: The CCD image shown here is a $5 \mathrm{~min} I$ band exposure obtained with EFOSC2 and the MPI-ESO $2.2 \mathrm{~m}$ telescope in April 1992. The field is heavily reddened. Object A is a G-K type star. RX J1749.8-3312 = 1RXS J174948.4-331215 is probably identified with the soft transient SLX 1746-331 (Skinner et al. 1990). The source was discovered by the Spacelab-2 coded-mask X-ray telescope in July-August 1985. Based on its ultrasoft spectrum and transient nature White \& van Paradijs (1996) classify the source as a black hole transient. During the ROSAT survey observation (1990 Sep. 08.97 to 1990 Sep. 10.30) the source was caught in outburst and not detected during a subsequent ROSAT HRI follow-up observation (1994 Oct. 02.25 - 02.27). Considering the expected low mass of the companion star and high interstellar absorption on the line of sight, the optical identification is likely to be difficult outside X-ray outbursts.

RX J1804.1+0042 = PMM0900-11515260: Follow-up ROSAT HRI observations show the source to be extended. Its mean position is $\mathrm{RA}=180408.6, \mathrm{DEC}=+004226.3$, well centered on an extended optical object which could be an elliptical galaxy. A low $\mathrm{S} / \mathrm{N}$ spectrum suggests a possible redshift of $z=0.07 \pm 0.02$. The galaxy is 
probably identified with the Parkes-MIT-NRAO radio source PMN J1804+0042 (Brinkmann et al. 1997).

RX J1936.3+2632 = A: $=2$ EUVE J1936+26.5

RX J1943.9+2118 = ??: We show a 10 min $I$ band CCD exposure obtained in September 1995 with the $1.2 \mathrm{~m}$ telescope at OHP. The large error circle represents the RGPS localisation whereas the small one is derived from a followup ROSAT HRI observation. The HRI position is RA = 194356.16 , DEC $=+211824.8$ with a conservative error circle of $10^{\prime \prime}$. A is a reddened object without marked features apart from the $\mathrm{Na}$ I line and a possible $\mathrm{Mg} \mathrm{G}$ band hollow. The position of $\mathrm{A}, \mathrm{RA}=194356.2, \mathrm{DEC}=$ +211820.4 is outside the error circle of the radio source NVSS J194356+211826 which is itself close to the centre of the HRI error circle. The overall radio, optical and X-ray picture favours a heavily absorbed extragalactic identification.

RX $\mathbf{J 1 9 5 0 . 0 + 3 8 2 1}=$ ??: Objects A, B, C and D are $\mathrm{G}$ type stars. The RGPS error circle contains a radio source NVSS J195004+382210 which is located $\approx 8^{\prime \prime}$ north of A. RX J2102.0+3359 = ?? UV excess object: We show here the BRASS position overlayed on a $10 \mathrm{~min} B$ filter CCD image obtained with the OHP $1.2 \mathrm{~m}$ telescope in September 1995. Object A is a late G early K star, B a $\mathrm{G}$ star. A low $\mathrm{S} / \mathrm{N}$ spectrum reveals a featureless continuum for object C. Object X, located at RA $=210201.4$, DEC $=+335929.6$ exhibits a strong UV excess with $B=21.6$ and $U-B=-1.1 \pm 0.3$. The softness of the $\mathrm{X}$-ray source and the presence of an UV excess object in the ROSAT error circle argue in favour of an AM Her system. Final identification should however await spectroscopic observations.

RX J2104.2+2118 = ??: Objects A and B are late type stars. Object $\mathrm{E}$ displays a blue featureless continuum. The radio source NVSSJ210415 +211805 , is located in the RGPS and BRASS error circles, $\approx 2^{\prime \prime}$ southern of object F.

RX J2156.3+3318 = PMM1200-18997159: X-ray emission is slightly extended by 1 arcmin and centered on the presumably elliptical galaxy PMM1200-18997159 ( $z=$ $0.079, B=16.2, R=12.4)$. The $F_{\mathrm{X}} / F_{\text {opt }}$ ratio is compatible with normal emission from the galaxy without the need to add cluster contribution. The radio source NVSS $\mathrm{J} 215623+331837$ is coincident with the galaxy.

Note added in proof: While the paper was under revision we obtained new observations of the field of RX J0759+0748 revealing that object D is an AGN at $z$ $\approx 0.15$

Acknowledgements. We thank the night assistants at Observatoire de Haute-Provence for carrying out some of the observations at the $1.2 \mathrm{~m}$ telescope. We are grateful to the referee for many helpful comments and suggestions. The ROSAT project is supported by the Bundesministerium für Bildung, Wissenschaft, Forschung und Technologie (BMBF/DLR) and the Max-Planck-Gesellschaft. C.M. acknowledges support from a CNRS-MPG cooperation contract and thanks Prof. J. Trümper and the ROSAT group for their hospitality and fruitful discussions. We are particularly grateful to the MPE team for providing early access to RASS data. This research has made use of the ALADIN sky atlas and of the SIMBAD database operated at CDS, Strasbourg, France. We thank F. Ochsenbein for providing easy access to the GSC and to the USNO-A1.0 catalogues. Finding charts were extracted from the Digitized Sky Survey, produced at the Space Telescope Science Institute (ST ScI) under U.S. Government grant NAG $\mathrm{W}-2166$.

\section{References}

Banse K., Crane P., Ounnas C., Ponz D., 1983, "MIDAS" in Proc. of DECUS, Zurich, p. 87

Barret D., Motch C., Pietsch W., Voges W., 1995, A\&A 296, 459

Beuermann K., Schwope A.D., 1994, in: Interacting binary stars, ASP Conf. Ser. 56, 119

Beuermann K., Reinsch K., Barwig H., et al., 1995, A\&A 294, L1

Bonnarel F., Ziaeepour H, Bartlett J.G., et al., 1997, IAU 179 Symposium Proceedings. Brain McLean, p. 469

Brinkmann W., Siebert J., Feigelson E.D., et al., 1997, A\&A 323,739

Burwitz V., Reinsch K., Schwope A.D., et al., 1996, A\&A 305, 507

Dickey J.M., Lockman F.J., 1990, ARA\&A 28, 215

Greiner J., Remillard R., Motch C., 1995, IUA Circ. No. 6200

Greiner J., Danner R., Bade N., Richter G.A., Kroll P., Komossa S., 1996, A\&A 310, 384

Griffith M.R., Wright A.E., Burke B.F., Ekers R.D., 1995, ApJS 97, 347

Guillout P., Haywood M., Motch C., Robin A.C., 1996a, MPE Report 263, 41

Guillout P., Haywood M., Motch C., Robin A.C., 1996b, A\&A 316,89

Haberl F., Motch C., 1995, A\&A 297, L37

Haberl F., Motch C., Buckley D.A.H., Zickgraf F-J., Pietsch W., 1997, A\&A 326, 662

Hausschildt M., 1987, A\&A 184, 43

Hertz P., Grindlay J.E., 1984, ApJ 278, 137

Jacoby G.H., Hunter D.A., Christian C.A., 1984, ApJS 56, 257

Jaschek C., Jaschek M., 1987, "The Classification of Stars", Press Syndicate of the University of Cambridge (U.S.A.)

Kock A., Meisenheimer K., Brinkmann W., Neumann M., Siebert J., 1996, A\&A 307, 745

Lasker B.M., Sturch C.R., McLean B.J., et al., 1990, AJ 99, 2019

Lemaitre G., Kohler D., Lacroix D., Meunier J.P., Vin A., 1990, A\&A 228, 546

Maccacaro T., Feigelson E.D., Fener M., et al., 1982, ApJ 253, 504

Malina R.F., Marshall H.L., Antia B., et al., 1994, AJ 107, 751

Mason K.O., Watson M.G., Ponman T.J., et al., 1992, MNRAS 258,749

Mason K.O., Hassal B.J.M., Bromage G., et al., 1995, MNRAS 274,1194

Monet D.G., et al., 1997, Publication of the US Naval Observatory Flagstaff Station 
Motch C., 1991a, MPE Report MB-ROS-ME-MA00/181

Motch C., Belloni T., Buckley D., et al., 1991b, A\&A 246, L24

Motch C., Werner K., Pakull M.W., 1993, A\&A 268, 561

Motch C., Hasinger G., Pietsch W., 1994, A\&A 284, 827

Motch C., Haberl F., 1995, in proceedings of the Cape Workshop on Magnetic Cataclysmic Variables, ASP Conf. Ser., Buckley D.A.H. and Warner B. (eds.), p. 109

Motch C., Haberl F., Guillout P., Pakull, et al., 1996, A\&A 307,459

Motch C., Guillout P., Haberl F., et al., 1997a, A\&A 318, 111

Motch C., Haberl F., Dennerl K., Pakull M., Janot-Pacheco E., 1997b, A\&A 323, 853

Ögelman H., Orio M., Krautter J., Starfield S., 1993, Nat 361, 331

Pavlinsky M.N., Grebenev S.A., Sunyaev R.A., 1994, ApJ 425, 110

Pfeffermann E., Briel U.G., Hippmann H., et al., 1986, SPIE 733,519

Pounds K.A., Allan D.J., Barber C., 1993, MNRAS 260, 77P

Pye J.P., Mc Gale P.A., Allan D.J., et al., 1995, MNRAS 274, 1165

Remillard R.A., Bradt H.V.D., Brissenden R.J.V., et al., 1993, AJ 105, 2089

Russel J.L., Lasker B.M., McLean B.J., Sturch C.R., Jenkner H., 1990, AJ 99, 2059
Schachter J.F., Remillard R., Saar S., et al., 1996, ApJ 463, 747

Skinner G.K., Foster A.J., Willmore A.P., Eyles C.J., 1990, MNRAS 243, 72

Sterzik M.F., Alcala J.M., Neuhäuser R., Schmitt J.H.M.M., 1995, A\&A 297, 418

Turnshek D.E., Turnshek D.A., Craine E.R., Boeshaar P.C., 1985, "An Atlas of Digital Spectra of Cool Stars", Western Research Company, Astronomy and Astrophysics Series, Volume 1

Vennes S., Christian D.J., Mathioudakis M., Doyle J.G., 1997, A\&A 318, L9

Vigotti M., Grueff G., Perley R., Clark B.G., Bridle A.H., 1989 AJ 98, 419

Voges W., 1992, in Proceedings of Satellite Symposium 3, "International Space Year Conference", ESA ISY-3, 9

Voges W., Gruber R., Paul J., et al., 1992, in Proceedings of Satellite Symposium 3, "International Space Year Conference", ESA ISY-3, 1

Voges W., Aschenbach B., Boller T., et al., 1996, IUA Circ. No. 6420

Walter F.M., Wolk S.J., Neuhäuser R., 1996, Nat 379, 18

White N.E., van Paradijs J., 1996, ApJ 473, L29

Zickgraf F.-J., Thiering I., Krautter J., et al., 1997, A\&AS 123, 103 
Table 4. X-ray characteristics of ROSAT survey sources derived from the SASS as in October 1991. Coordinates are equinox 2000.0. Column (2) indicates the source selection origin as shown in Fig. 6, S, soft, A, absorbed soft, X, X-ray binary like, i.e. hard. Remaining sources are split in $\mathrm{B}$, bright (count rates $\geq 0.25 \mathrm{cts} \mathrm{s}^{-1}$ ) and $\mathrm{O}$, others. Whenever the SASS-I RGPS source is bright enough to appear in the BRASS catalogue we give the 1RXS name

\begin{tabular}{|c|c|c|c|c|c|c|c|c|c|c|c|}
\hline $\begin{array}{l}\text { Source } \\
\text { Name }\end{array}$ & Sel & $\begin{array}{c}\text { Right } \\
\text { Ascension }\end{array}$ & Declination & $\begin{array}{c}r_{90} \\
\prime \prime\end{array}$ & $\begin{array}{l}\text { Cnt rate } \\
\text { cts s }^{-1}\end{array}$ & $\begin{array}{c}\text { Error } \\
\operatorname{cts~s}^{-1}\end{array}$ & $H R 1$ & Error & $H R 2$ & Error & $\begin{array}{c}\text { BRASS } \\
\text { Name }\end{array}$ \\
\hline RX J0035.8+5950 & $\mathrm{X}$ & 003552.6 & 595008 & 19.2 & 0.337 & 0.037 & 0.91 & 0.06 & 0.33 & 0.08 & 1RXS J003552.8+595006 \\
\hline RX J0119.0+7033 & $\mathrm{X}$ & 011902.8 & 703309 & 25.9 & 0.145 & 0.020 & 0.93 & 0.07 & 0.43 & 0.14 & 1RXS J011859.8+703336 \\
\hline RX J0143.7+6349 & A & 014344.0 & 634930 & 21.5 & 0.051 & 0.013 & 0.02 & 0.53 & -1.00 & * & 1RXS J014343.7+634931 \\
\hline RX J0146.0+6348 & A & 014605.8 & 634852 & 21.5 & 0.054 & 0.017 & 0.04 & 0.71 & -1.00 & $*$ & 1RXS J014605.5+634853 \\
\hline RX J0202.2+6934 & $\mathrm{O}$ & $\begin{array}{lll}02 & 02 & 14.7\end{array}$ & 693423 & 21.5 & 0.042 & 0.010 & 0.07 & 0.52 & -0.15 & 0.52 & \\
\hline RX J0222.1+5221 & $\mathrm{X}$ & $02 \quad 2206.9$ & 522111 & 25.9 & 0.174 & 0.028 & 0.86 & 0.13 & 0.50 & 0.15 & 1RXS J022206.0+522112 \\
\hline RX J0222.5+5033 & $\mathrm{B}$ & 022235.0 & 503334 & 18.4 & 0.422 & 0.034 & -0.07 & 0.08 & -0.28 & 0.11 & 1RXS J022234.1+503335 \\
\hline RX J0254.6+3931 & $\mathrm{O}$ & 025441.8 & 393141 & 20.3 & 0.121 & 0.020 & 1.00 & * & -0.35 & 0.16 & 1RXS J025441.4+393143 \\
\hline RX J0256.3+6141 & A & $\begin{array}{lll}02 & 56 & 20.7\end{array}$ & 614124 & 21.5 & 0.153 & 0.021 & -0.01 & 0.14 & -0.61 & 0.32 & 1RXS J025620.0+614129 \\
\hline RX J0324.7+3410 & $\mathrm{X}$ & 032442.2 & 341051 & 21.5 & 0.235 & 0.026 & 0.91 & 0.08 & 0.02 & 0.11 & 1RXS J032441.3+341056 \\
\hline RX J0325.2+4042 & $\mathrm{O}$ & 032517.6 & 404200 & 27.5 & 0.045 & 0.013 & 0.45 & 0.42 & -0.01 & 0.53 & \\
\hline RX J0337.0+4738 & $\mathrm{X}$ & 033704.0 & 473853 & 21.5 & 0.134 & 0.021 & 0.83 & 0.16 & 0.13 & 0.17 & 1RXS J033703.9+473852 \\
\hline RX J0416.0+5237 & A & 041603.0 & 523731 & 22.9 & 0.102 & 0.025 & -0.24 & 0.28 & -0.60 & 0.45 & 1RXS J041602.7+523737 \\
\hline RX J0434.7+4014 & $\mathrm{X}$ & 043442.2 & 401421 & 19.2 & 0.197 & 0.024 & 0.84 & 0.15 & 0.22 & 0.13 & 1RXS J043442.1+401425 \\
\hline RX J0452.0+4932 & $\mathrm{X}$ & 045205.0 & 493246 & 17.7 & 0.595 & 0.048 & 0.92 & 0.07 & 0.42 & 0.06 & 1RXS J045205.0+493248 \\
\hline RX J0459.8+1808 & $\mathrm{O}$ & 045951.2 & 180847 & 38.5 & 0.061 & 0.014 & 0.60 & 0.33 & 0.10 & 0.26 & \\
\hline RX J0508.3+1721 & $\mathrm{X}$ & 050820.6 & 172138 & 22.9 & 0.064 & 0.018 & 0.89 & 0.10 & 0.47 & 0.40 & 1RXS J050820.9+172134 \\
\hline RX J0508.9+2113 & $\mathrm{X}$ & $\begin{array}{lll}05 & 08 & 55.7\end{array}$ & 211304 & 31.0 & 0.058 & 0.018 & 0.88 & 0.12 & -0.02 & 0.18 & 1RXS J050855.3+2 \\
\hline RX J0529.0+0934 & $\mathrm{X}$ & $\begin{array}{lll}05 & 29 & 03.1\end{array}$ & 093438 & 18.4 & 0.431 & 0.042 & 0.96 & 0.04 & 0.26 & 0.07 & 1RXS J052902.7+093439 \\
\hline RX J0535.0+6450 & $\mathrm{S}$ & 053500.7 & 645047 & 25.9 & 0.160 & 0.034 & -0.75 & 0.22 & 0.11 & 0.70 & 1RXS J053501.1+645046 \\
\hline RX J0554.7+1055 & A & 055445.8 & 105559 & 21.5 & 0.193 & 0.033 & -0.16 & 0.12 & -0.69 & 0.27 & 1RXS J055446.0+105559 \\
\hline RX J0602.1+2828 & $\mathrm{X}$ & $\begin{array}{lll}06 & 02 & 10.6\end{array}$ & 282825 & 18.4 & 0.708 & 0.057 & 0.97 & 0.03 & 0.23 & 0.06 & 1RXS J060210.7+282821 \\
\hline RX J0602.2+2837 & $\mathrm{X}$ & $\begin{array}{lll}06 & 02 & 17.5\end{array}$ & 283704 & 25.9 & 0.149 & 0.031 & 0.93 & 0.06 & -0.05 & 0.13 & \\
\hline RX J0608.0+3058 & $\mathrm{X}$ & $\begin{array}{lll}06 & 08 & 01.7\end{array}$ & 305853 & 22.9 & 0.112 & 0.025 & 0.78 & 0.19 & 0.46 & 0.40 & 1RXS J060801.7+305847 \\
\hline RX J0620.6+2644 & $\mathrm{X}$ & 062040.3 & 264428 & 25.9 & 0.338 & 0.038 & 0.93 & 0.07 & 0.06 & 0.13 & 1RXS J062040.0+2 \\
\hline RX J0621.2+4415 & A & 062113.8 & 441547 & 21.5 & 0.510 & 0.074 & 0.04 & 0.14 & -0.43 & 0.19 & 1RXS J062113.1+4 \\
\hline RX J0621.7+1747 & $\mathrm{X}$ & 062147.8 & 174730 & 19.2 & 0.113 & 0.021 & 0.84 & 0.15 & 0.30 & 0.46 & 1RXS J062148.1+174736 \\
\hline RX J0625.8-0101 & $\mathrm{S}$ & 062549.8 & -010140 & 21.5 & 0.143 & 0.022 & -0.46 & 0.16 & -0.06 & 0.53 & 1RXS J062549.8-010138 \\
\hline RX J0635.9+0755 & $\mathrm{X}$ & 063558.2 & 075528 & 20.3 & 0.247 & 0.029 & 0.81 & 0.17 & 0.26 & 0.11 & 1RXS J063558.5+075527 \\
\hline RX J0648.7+1516 & $\mathrm{X}$ & $06 \quad 48 \quad 47.4$ & 151626 & * & 0.679 & 0.679 & 1.03 & 0.06 & 0.03 & 0.06 & 1RXS J064847.8+151626 \\
\hline RX J0649.8-0737 & $\mathrm{S}$ & $0649 \quad 50.2$ & -073741 & 21.5 & 0.557 & 0.055 & -0.69 & 0.08 & -0.55 & 0.36 & 1RXS J064949.8-073734 \\
\hline RX J0656.8-1424 & $\mathrm{X}$ & 065653.6 & -142456 & 17.7 & 0.788 & 0.048 & 0.80 & 0.05 & 0.07 & 0.06 & 1RXS J065653.2-142455 \\
\hline RX J0702.0+1257 & $\mathrm{S}$ & $\begin{array}{lll}07 & 02 & 04.1\end{array}$ & 125750 & 18.4 & 1.180 & 0.067 & -0.67 & 0.04 & -0.28 & 0.15 & 1RXS J070204.3+1 \\
\hline RX J0704.5-0612 & $\mathrm{X}$ & 070431.3 & $-06 \quad 12 \quad 35$ & 24.3 & 0.113 & 0.021 & 0.78 & 0.20 & -0.03 & 0.18 & 1RXS J070431.1- \\
\hline RX J0713.1-0511 & B & 071311.1 & -051152 & 17.7 & 1.470 & 0.069 & -0.23 & 0.05 & -0.31 & 0.07 & 1RXS J071311.0-( \\
\hline RX J0717.4-1119 & $\mathrm{X}$ & 071725.1 & $-11 \quad 1943$ & 27.5 & 0.145 & 0.026 & 0.83 & 0.16 & 0.45 & 0.13 & 1RXS J071722.6-111954 \\
\hline RX J0721.3-5720 & $\mathrm{O}$ & 072122.8 & -572049 & 32.8 & 0.199 & 0.020 & -0.01 & 0.10 & -0.12 & 0.13 & 1RXS J072123.9-572034 \\
\hline RX J0749.1-0549 & $\mathrm{S}$ & $\begin{array}{lll}07 & 49 & 09.7\end{array}$ & -054931 & 27.5 & 0.615 & 0.082 & -0.80 & 0.18 & -0.69 & 0.37 & 1RXS J074909.8-054933 \\
\hline RX J0750.9+0320 & B & 075059.8 & 032018 & 18.4 & 0.808 & 0.057 & 0.32 & 0.07 & -0.10 & 0.09 & 1RXS J075100.0+032017 \\
\hline RX J0755.7-0157 & $\mathrm{B}$ & 075547.3 & -015748 & 17.7 & 0.882 & 0.058 & 0.29 & 0.07 & -0.16 & 0.09 & 1RXS J075547.4-015742 \\
\hline RX J0759.1+0748 & A & 075909.0 & 074826 & 21.5 & 3.360 & 0.200 & -0.16 & 0.06 & -0.43 & 0.09 & 1RXS J075908.8+0 \\
\hline RX J0801.9-4946 & $\mathrm{X}$ & 080157.8 & -494642 & 20.3 & 0.173 & 0.018 & 0.92 & 0.07 & 0.32 & 0.10 & 1RXS J080157.7- \\
\hline RX J0811.1-5555 & $\mathrm{O}$ & 081109.8 & -555526 & 36.5 & 0.125 & 0.014 & 0.18 & 0.11 & 0.34 & 0.16 & 1RXS J081108.7-5 \\
\hline RX J0816.4-1311 & B & $\begin{array}{llll}08 & 16 & 26.7\end{array}$ & -131146 & 19.2 & 0.861 & 0.058 & 0.92 & 0.03 & -0.13 & 0.08 & 1RXS J081626.9-131149 \\
\hline RX J0818.9-2252 & $\mathrm{X}$ & $08 \quad 18 \quad 57.3$ & $-22 \quad 5232$ & 24.3 & 0.566 & 0.047 & 0.90 & 0.07 & 0.03 & 0.08 & 1RXS J081858.0-225229 \\
\hline RX J0819.2-0756 & A & $\begin{array}{lll}08 & 19 & 17.7\end{array}$ & -075620 & 20.3 & 0.158 & 0.029 & 0.83 & 0.16 & -0.45 & 0.18 & 1RXS J081917.6-075620 \\
\hline RX J0828.5-5138 & $\mathrm{O}$ & 082833.2 & -513819 & 25.9 & 0.236 & 0.021 & 0.11 & 0.10 & -0.11 & 0.11 & 1RXS J082832.2-513828 \\
\hline RX J0845.7-3544 & A & $0845 \quad 43.0$ & -354427 & 22.9 & 0.077 & 0.016 & 0.34 & 0.46 & -0.70 & 0.26 & \\
\hline RX J0856.4-2241 & A & 085626.4 & -224142 & 25.9 & 0.102 & 0.025 & 0.30 & 0.48 & -0.49 & 0.40 & 1RXS J085626.3-224141 \\
\hline RX J0913.0-2103 & B & 091300.8 & -210323 & 17.7 & 0.924 & 0.052 & 0.94 & 0.02 & -0.14 & 0.07 & 1RXS J091300.4-210315 \\
\hline RX J0935.5-2802 & B & 093531.5 & -280250 & 21.5 & 0.267 & 0.031 & -0.04 & 0.11 & -0.26 & 0.17 & 1RXS J093530.9-280255 \\
\hline RX J1023.9-4336 & B & 102355.5 & -433603 & 19.2 & 1.280 & 0.062 & 0.92 & 0.03 & -0.18 & 0.05 & 1RXS J102356.4-433602 \\
\hline RX J1155.4-5641 & $\mathrm{B}$ & 115527.7 & -564159 & 31.0 & 0.819 & 0.067 & 0.65 & 0.05 & 0.10 & 0.06 & 1RXS J115527.6-564149 \\
\hline RX J1606.6-4618 & $\mathrm{X}$ & 160637.1 & $-4618 \quad 30$ & 31.0 & 0.139 & 0.025 & 0.84 & 0.15 & 0.13 & 0.17 & 1RXS J160637.1-461835 \\
\hline RX J1639.7-3920 & $\mathrm{X}$ & 163947.8 & -392015 & 31.0 & 0.254 & 0.033 & 0.80 & 0.18 & 0.37 & 0.13 & 1RXS J163947.8-392023 \\
\hline RX J1704.3-4020 & $\mathrm{X}$ & 170419.4 & -402013 & 21.5 & 0.214 & 0.039 & 0.88 & 0.12 & 0.36 & 0.13 & 1RXS J170419.7-402012 \\
\hline RX J1718.4-4029 & $\mathrm{X}$ & 171824.2 & -402934 & 31.0 & 0.167 & 0.039 & 0.74 & 0.23 & 0.63 & 0.31 & 1RXS J171824.2-402934 \\
\hline RX J1739.4-2942 & $\mathrm{X}$ & 173930.1 & -294207 & 19.2 & 0.154 & 0.030 & 0.85 & 0.14 & 0.84 & 0.15 & 1RXS J173930.3-294211 \\
\hline RX J1740.7-2818 & $\mathrm{X}$ & 174042.8 & -281803 & 20.3 & 0.336 & 0.041 & 0.83 & 0.15 & 0.85 & 0.14 & 1RXS J174043.1-281806 \\
\hline RX J1741.4+0348 & $\mathrm{X}$ & 174127.5 & 034848 & 18.4 & 1.180 & 0.063 & 0.91 & 0.09 & 0.02 & 0.05 & 1RXS J174128.1+034848 \\
\hline RX J1742.3-2737 & $\mathrm{X}$ & 174220.2 & -273736 & 24.3 & 0.166 & 0.033 & 0.90 & 0.10 & 0.74 & 0.22 & 1RXS J174220.8-273736 \\
\hline RX J1749.8-3312 & $\mathrm{X}$ & 174948.3 & $-3312 \quad 26$ & 18.4 & 0.543 & 0.062 & 0.97 & 0.03 & 0.41 & 0.07 & 1RXS J174948.4-331215 \\
\hline
\end{tabular}


Table 4. continued

\begin{tabular}{|c|c|c|c|c|c|c|c|c|c|c|c|}
\hline $\begin{array}{c}\text { Source } \\
\text { Name }\end{array}$ & Sel & $\begin{array}{c}\text { Right } \\
\text { Ascension }\end{array}$ & Declination & $\begin{array}{c}r_{90} \\
\prime \prime\end{array}$ & $\begin{array}{l}\text { Cnt rate } \\
\text { cts s}^{-1}\end{array}$ & $\begin{array}{c}\text { Error } \\
\text { cts s }^{-1}\end{array}$ & $H R 1$ & Error & $H R 2$ & Error & $\begin{array}{c}\text { BRASS } \\
\text { Name }\end{array}$ \\
\hline RX J1804.1+0042 & $\mathrm{X}$ & 180408.3 & 004229 & 22.9 & 0.354 & 0.042 & 0.95 & 0.05 & 0.25 & 0.09 & 1RXS J180408.7+004229 \\
\hline RX J1925.0+4429 & A & 192501.8 & 442943 & 21.5 & 0.317 & 0.030 & 0.51 & 0.08 & -0.60 & 0.08 & 1RXS J192502.2+442948 \\
\hline RX J1929.8+4622 & A & 192950.1 & 462216 & 21.5 & 0.392 & 0.030 & 0.59 & 0.09 & -0.41 & 0.07 & 1RXS J192949.7+462231 \\
\hline RX J1931.1+0937 & $\mathrm{X}$ & 193109.0 & 093722 & 17.7 & 0.625 & 0.047 & 0.93 & 0.07 & 0.25 & 0.08 & 1RXS J193109.5+093714 \\
\hline RX J1935.4+3746 & A & 193529.4 & 374609 & 25.9 & 0.528 & 0.042 & -0.13 & 0.08 & -0.45 & 0.12 & 1RXS J193528.9+374605 \\
\hline RX J1936.3+2632 & $\mathrm{S}$ & 193618.1 & 263242 & 31.0 & 0.100 & 0.022 & -0.64 & 0.30 & -0.64 & 0.42 & \\
\hline RX J1943.9+2118 & $\mathrm{X}$ & 194355.4 & 211815 & 32.8 & 0.103 & 0.023 & 0.82 & 0.17 & 0.90 & 0.09 & 1RXS J194356.1+211731 \\
\hline RX J1946.2-0444 & A & 194616.5 & -044447 & 21.5 & 0.114 & 0.026 & 1.00 & * & -0.46 & 0.18 & 1RXS J194616.9-044456 \\
\hline RX J1947.3+3045 & $\mathrm{S}$ & 194723.4 & 304555 & 19.2 & 1.170 & 0.064 & -0.96 & 0.03 & -0.18 & 0.68 & 1RXS J194723.8+304558 \\
\hline RX J1950.0+3821 & A & 195004.7 & 382152 & 20.3 & 0.117 & 0.023 & 0.92 & 0.08 & -0.44 & 0.16 & 1RXS J195005.2+382155 \\
\hline RX J1951.7+3716 & A & 195146.2 & 371656 & 27.5 & 0.102 & 0.023 & 0.89 & 0.11 & -0.42 & 0.19 & 1RXS J195148.7+371712 \\
\hline RX J1953.6+5025 & $\mathrm{O}$ & 195341.0 & 502503 & 22.9 & 0.203 & 0.018 & 0.11 & 0.09 & 0.01 & 0.13 & 1RXS J195340.6+502456 \\
\hline RX J1956.7+5304 & $\mathrm{O}$ & 195646.7 & 530431 & 31.0 & 0.170 & 0.014 & 0.45 & 0.08 & -0.26 & 0.10 & 1RXS J195646.5+530424 \\
\hline RX J2002.1+5438 & B & 200207.9 & 543805 & 31.0 & 0.435 & 0.023 & 0.27 & 0.05 & -0.25 & 0.06 & 1RXS J200208.2+543827 \\
\hline RX J2010.5+0632 & B & 201034.3 & 063206 & 21.5 & 0.881 & 0.070 & -0.20 & 0.08 & -0.21 & 0.11 & 1RXS J201034.8+063208 \\
\hline RX J2014.8+4501 & B & 201449.8 & 450132 & 22.9 & 0.610 & 0.030 & 0.46 & 0.05 & -0.07 & 0.06 & 1RXS J201449.4+450143 \\
\hline RX J2019.8+2256 & A & 201948.9 & 225621 & 18.4 & 0.523 & 0.037 & -0.21 & 0.08 & -0.44 & 0.11 & 1RXS J201949.3+225628 \\
\hline RX J2021.7+5213 & A & 202144.2 & 521354 & 25.9 & 0.071 & 0.011 & 0.03 & 0.37 & -0.59 & 0.27 & 1RXS J202144.2+521348 \\
\hline RX J2033.4+3128 & B & 203325.3 & 312803 & 20.3 & 0.273 & 0.026 & 0.26 & 0.10 & 0.02 & 0.13 & 1RXS J203324.9+312816 \\
\hline RX J2040.3+1059 & B & 204018.5 & 105940 & 19.2 & 0.532 & 0.052 & 0.45 & 0.09 & -0.05 & 0.11 & 1RXS J204019.1+105941 \\
\hline RX J2043.9+5314 & $\mathrm{X}$ & 204359.4 & 531429 & 21.5 & 0.229 & 0.018 & 0.95 & 0.04 & 0.47 & 0.07 & 1RXS J204400.1+531434 \\
\hline RX J2044.0+2833 & B & 204403.9 & 283305 & 19.2 & 0.303 & 0.030 & 0.91 & 0.06 & -0.24 & 0.09 & 1RXS J204404.0+283303 \\
\hline RX J2102.0+3359 & $\mathrm{S}$ & 210202.9 & 335922 & 24.3 & 0.160 & 0.019 & -0.66 & 0.12 & -0.76 & 0.18 & 1RXS J210201.7+335932 \\
\hline RX J2102.6+4553 & A & 210240.5 & 455305 & 24.3 & 0.216 & 0.022 & -0.12 & 0.10 & -0.68 & 0.19 & 1RXS J210241.4+455305 \\
\hline $\mathrm{RX} \mathrm{J} 2104.2+2118$ & $\mathrm{X}$ & 210416.2 & 211820 & 20.3 & 0.145 & 0.019 & 0.88 & 0.11 & 0.19 & 0.15 & 1RXS J210416.4+211816 \\
\hline RX J2109.7+4029 & $\mathrm{X}$ & 210947.3 & 402947 & 31.0 & 0.121 & 0.018 & 0.82 & 0.17 & 0.21 & 0.14 & 1RXS J210948.0+402944 \\
\hline RX J2133.7+5107 & B & 213343.5 & $\begin{array}{lll}5107 & 20\end{array}$ & 19.2 & 0.513 & 0.031 & 0.90 & 0.06 & -0.16 & 0.06 & 1RXS J213344.1+510725 \\
\hline RX J2155.3+5938 & $\mathrm{S}$ & 215521.0 & 593849 & 34.7 & 0.112 & 0.024 & -0.41 & 0.18 & -0.59 & 0.32 & 1RXS J215522.8+593843 \\
\hline RX J2156.3+3318 & B & 215623.3 & 331840 & 22.9 & 0.516 & 0.042 & 0.99 & 0.01 & -0.23 & 0.09 & 1RXS J215623.8+331829 \\
\hline RX J2255.0+5540 & $\mathrm{X}$ & 225503.3 & 554053 & 24.3 & 0.103 & 0.026 & 0.75 & 0.22 & 0.04 & 0.17 & 1RXS J225504.1+554052 \\
\hline RX J2322.6+6113 & $\mathrm{X}$ & 232238.3 & $61 \quad 1329$ & 34.7 & 0.118 & 0.023 & 0.87 & 0.10 & 0.06 & 0.20 & 1RXS J232241.3+611335 \\
\hline
\end{tabular}


Table 5. Optical identifications of ROSAT survey sources. Coordinates are equinox 2000.0. Column (2) indicates the source selection origin as shown in Fig. 6, and given in Table 4. Column (3) gives X-ray to optical distance in units of the $90 \%$ confidence radius. In column Class, "AC" stands for active corona and "Gal" for normal galaxy. The last column indicates whether a finding chart is provided

\begin{tabular}{|c|c|c|c|c|c|c|c|c|c|c|c|c|c|c|}
\hline $\begin{array}{l}\text { Source } \\
\text { Name }\end{array}$ & Sel & $\begin{array}{r}d(x-o) \\
\left(\mathrm{r}_{90}\right)\end{array}$ & $\begin{array}{l}\mathrm{R} \\
\mathrm{h}\end{array}$ & $\begin{array}{r}\text { ght } \\
\mathrm{m}\end{array}$ & $\begin{array}{r}\text { Asce. } \\
\text { s }\end{array}$ & d & $\begin{array}{c}\overline{\text { Dec }} \\
,\end{array}$ & "I & $\begin{array}{r}\text { Optical } \\
\text { Identification }\end{array}$ & $\bar{V}$ & $\bar{B}$ & Class & Type & $\begin{array}{c}\text { Finding } \\
\text { Chart }\end{array}$ \\
\hline RX J0035.8+5950 & $\mathrm{X}$ & & * & & & * & 水 & & $? ?$ & & * & UNID & & $\bar{Y}$ \\
\hline RX J0119.0+7033 & $\mathrm{X}$ & 0.64 & 1 & 19 & 3.32 & 70 & 33 & 25.7 & GSC0430101235 & 9.89 & $*$ & $\mathrm{AC}$ & $\mathrm{K} 1 \mathrm{~V}$ & \\
\hline RX J0143.7+6349 & A & 1.09 & 1 & 43 & 43.57 & 63 & 49 & 54.0 & HD 10436 & 8.41 & * & $\mathrm{AC}$ & K5V & \\
\hline RX J0146.0+6348 & $\mathrm{A}$ & 0.31 & 1 & 46 & 4.96 & 63 & 48 & 56.4 & HD 10663 & 8.69 & * & $\mathrm{AC}$ & G2V & \\
\hline RX J0202.2+6934 & $\mathrm{O}$ & 0.09 & 2 & 2 & 14.60 & 69 & 34 & 21.1 & GSC0431501020 & 11.72 & * & $\mathrm{AC}$ & $\mathrm{G} 2 \mathrm{~V}$ & \\
\hline RX J0222.1+5221 & $\mathrm{X}$ & 0.28 & 2 & 22 & 6.19 & 52 & 21 & 8.4 & PMM1350-02324516 & $*$ & 17.00 & $\mathrm{AGN}$ & Sy 1 & \\
\hline RX J0222.5+5033 & B & 0.91 & 2 & 22 & 33.42 & 50 & 33 & 41.9 & $\mathrm{BD}+49646$ & 10.10 & * & $\mathrm{AC}$ & G2V & \\
\hline RX J0254.6+3931 & $\mathrm{O}$ & 0.59 & 2 & 54 & 42.63 & 39 & 31 & 34.7 & CJ2 $0251+393$ & 16.10 & * & AGN & BLRG & \\
\hline RX J0256.3+6141 & A & 0.21 & 2 & 56 & 20.11 & 61 & 41 & 22.6 & GSC0404800621 & 13.08 & * & $\mathrm{AC}$ & $\mathrm{M} 2 \mathrm{Ve}$ & \\
\hline RX J0324.7+3410 & $\mathrm{X}$ & 0.66 & 3 & 24 & 41.16 & 34 & 10 & 45.9 & GSC0234901904 & 15.10 & $*$ & AGN & Sy 1 & \\
\hline RX J0325.2+4042 & $\mathrm{O}$ & 1.82 & 3 & 25 & 13.22 & 40 & 41 & 54.6 & PMM1275-02314407 & 15.20 & * & AGN & Sy 2: & \\
\hline RX J0337.0+4738 & $\mathrm{X}$ & 0.42 & 3 & 37 & 3.14 & 47 & 38 & 51.5 & PMM1350-03755404 & * & 17.00 & AGN & Sy 1 & \\
\hline RX J0416.0+5237 & $\mathrm{A}$ & 0.35 & 4 & 16 & 3.54 & 52 & 37 & 37.8 & GSC0371900494 & 11.70 & * & $\mathrm{AC}$ & $\mathrm{K} 7 \mathrm{~V}$ & \\
\hline RX J0434.7+4014 & $\mathrm{X}$ & 0.42 & 4 & 34 & 41.54 & 40 & 14 & 19.3 & PMM1275-03482924 & * & 15.20 & AGN & Sy 1 & \\
\hline RX J0452.0+4932 & $\mathrm{X}$ & 0.09 & 4 & 52 & 5.00 & 49 & 32 & 45.2 & H0432052 & 17.10 & * & AGN & Sy 1 & \\
\hline RX J0459.8+1808 & $\mathrm{O}$ & 0.34 & 4 & 59 & 51.90 & 18 & 8 & 38.9 & PMM1050-01591537 & * & 19.50 & $\mathrm{AGN}$ & Sy 1 & \\
\hline RX J0508.3+1721 & $\mathrm{X}$ & 0.71 & 5 & 8 & 19.72 & 17 & 21 & 48.1 & GSC0128601162 & 13.47 & * & AGN & Sy 2 : & \\
\hline RX J0508.9+2113 & $\mathrm{X}$ & 0.25 & 5 & 8 & 55.15 & 21 & 13 & 2.4 & PMM1050-01718259 & * & 17.70 & AGN & Sy 1 & \\
\hline RX J0529.0+0934 & $\mathrm{X}$ & $*$ & $*$ & * & * & * & * & * & $? ?$ & $*$ & $*$ & UNID & & $\mathrm{Y}$ \\
\hline RX J0535.0+6450 & $\mathrm{S}$ & 0.32 & 5 & 35 & 0.62 & 64 & 50 & 39.1 & GSC0408900790 & 12.37 & * & $\mathrm{AC}$ & $\mathrm{K}$ & \\
\hline RX J0554.7+1055 & A & 0.09 & 5 & 54 & 45.87 & 10 & 55 & 57.9 & GSC0072000052 & 11.93 & * & $\mathrm{AC}$ & G0V & \\
\hline RX J0602.1+2828 & $\mathrm{X}$ & 0.20 & 6 & 2 & 10.70 & 28 & 28 & 22.1 & PMM1125-03274684 & * & 15.00 & AGN & Sy 1 & \\
\hline RX J0602.2+2837 & $\mathrm{X}$ & $*$ & $*$ & * & $*$ & $*$ & $*$ & $*$ & $? ?$ & * & $*$ & UNID & & $\mathrm{Y}$ \\
\hline RX J0608.0+3058 & $\mathrm{X}$ & 0.64 & 6 & 8 & 0.95 & 30 & 58 & 42.0 & PMM1200-04416825 & * & 17.30 & AGN & Sy 1 & \\
\hline RX J0620.6+2644 & $\mathrm{X}$ & * & * & * & * & * & * & * & $? ?$ & * & * & UNID & & $\mathrm{Y}$ \\
\hline RX J0621.2+4415 & A & 3.05 & 6 & 21 & 12.50 & 44 & 14 & 43.4 & G $101-35$ & 11.93 & $*$ & $\mathrm{AC}$ & $\mathrm{M} 2 \mathrm{Ve}$ & \\
\hline RX J0621.7+1747 & $\mathrm{X}$ & * & * & * & * & * & * & * & $? ?$ & * & $*$ & UNID & & $\mathrm{Y}$ \\
\hline RX J0625.8-0101 & $\mathrm{S}$ & 0.34 & 6 & 25 & 49.51 & -1 & 1 & 46.3 & GSC0478501175 & 12.69 & * & $\mathrm{AC}$ & M1Ve & \\
\hline RX J0635.9+0755 & $\mathrm{X}$ & 0.35 & 6 & 35 & 58.28 & 7 & 55 & 21.0 & GSC0073302098 & 10.69 & * & $\mathrm{AC}$ & $\mathrm{G}-\mathrm{K}$ & \\
\hline RX J0648.7+1516 & $\mathrm{X}$ & $*$ & * & * & * & * & * & * & $? ?$ & * & * & UNID & & $\mathrm{Y}$ \\
\hline RX J0649.8-0737 & $\mathrm{S}$ & 0.49 & 6 & 49 & 50.90 & -7 & 37 & 40.2 & PMM0750-02887019 & * & 18.00 & $\mathrm{CV}$ & & $\mathrm{Y}$ \\
\hline RX J0656.8-1424 & $\mathrm{X}$ & 0.61 & 6 & 56 & 54.33 & -14 & 24 & 59.0 & GSC0539202173 & 9.77 & $*$ & $\mathrm{AC}$ & KoV & \\
\hline RX J0702.0+1257 & $\mathrm{S}$ & 0.29 & 7 & 2 & 3.99 & 12 & 57 & 55.4 & GSC0075701608 & 9.89 & * & $\mathrm{AC}$ & $\mathrm{Ke}$ & \\
\hline RX J0704.5-0612 & $\mathrm{X}$ & 0.75 & 7 & 4 & 31.46 & -6 & 12 & 17.2 & GSC0482603053 ?? & 13.00 & * & $\mathrm{AC} ? ?$ & $\mathrm{G}-\mathrm{K}$ & \\
\hline RX J0713.1-0511 & $\mathrm{B}$ & 0.53 & 7 & 13 & 11.22 & -5 & 11 & 43.4 & GSC0482302265 & 12.14 & * & $\mathrm{AC}$ & $\mathrm{M} 3 \mathrm{Ve}$ & \\
\hline RX J0717.4-1119 & $\mathrm{X}$ & * & $*$ & * & * & * & * & * & ?? & $*$ & $*$ & UNID & & Y \\
\hline RX J0721.3-5720 & $\mathrm{O}$ & 0.42 & 7 & 21 & 23.76 & -57 & 20 & 37.8 & GSC0855901016 comp & 11.23 & * & $\mathrm{AC}$ & $\mathrm{K} 0 \mathrm{~V}+\mathrm{M} 5 \mathrm{eV}$ & \\
\hline RX J0749.1-0549 & $\mathrm{S}$ & 0.47 & 7 & 49 & 10.45 & -5 & 49 & 24.9 & PMM0825-05603282 & * & 19.00 & $\mathrm{CV}$ & & $\mathrm{Y}$ \\
\hline RX J0750.9+0320 & B & 1.46 & 7 & 51 & 0.73 & 3 & 20 & 40.9 & PMM0900-05507668 & $*$ & 15.20 & AGN & Sy 1 & \\
\hline RX J0755.7-0157 & B & 0.36 & 7 & 55 & 47.32 & -1 & 57 & 41.7 & PMM0825-05763804 & $*$ & 14.80 & $\mathrm{AGN}$ & Sy 1 & \\
\hline RX J0759.1+0748 & $\mathrm{A}$ & * & $*$ & $*$ & $*$ & $*$ & $*$ & * & $? ?$ & $*$ & * & UNID & & $\mathrm{Y}$ \\
\hline RX J0801.9-4946 & $\mathrm{X}$ & 0.33 & 8 & 1 & 58.00 & -49 & 46 & 36.0 & ESO $209-12$ & 11.07 & * & AGN & Sa Sy 1 & \\
\hline RX J0811.1-5555 & $\mathrm{O}$ & 0.82 & 8 & 11 & 9.37 & -55 & 55 & 56.5 & GSC0857001980 & 11.97 & $*$ & $\mathrm{AC}$ & $\mathrm{G}-\mathrm{K} 0$ & \\
\hline RX J0816.4-1311 & B & 0.51 & 8 & 16 & 27.19 & -13 & 11 & 52.6 & PMM0750-06007988 & * & 17.40 & AGN & BL LAC & \\
\hline RX J0818.9-2252 & $\mathrm{X}$ & 0.28 & 8 & 18 & 57.70 & -22 & 52 & 35.9 & PMM0600-05866078 & * & 15.50 & AGN & Sy 1 & \\
\hline RX J0819.2-0756 & $\mathrm{A}$ & $*$ & $*$ & $*$ & $*$ & $*$ & $*$ & $*$ & $? ?$ & * & $*$ & UNID & & Y \\
\hline RX J0828.5-5138 & $\mathrm{O}$ & 0.58 & 8 & 28 & 32.34 & -51 & 38 & 32.6 & GSC0816200330 & 10.87 & $*$ & $\mathrm{AC}$ & $\mathrm{G}$ & \\
\hline RX J0845.7-3544 & A & 0.48 & 8 & 45 & 42.51 & -35 & 44 & 36.6 & GSC0714900583 & 12.09 & $*$ & $\mathrm{AC}$ & $\mathrm{G}-\mathrm{K}$ & \\
\hline RX J0856.4-2241 & A & 0.10 & 8 & 56 & 26.41 & -22 & 41 & 39.8 & GSC0658500334 & 12.41 & $*$ & $\mathrm{AC}$ & $\mathrm{M} 0-1 \mathrm{~V}(\mathrm{e})$ & \\
\hline RX J0913.0-2103 & B & 0.51 & 9 & 13 & 0.17 & -21 & 3 & 20.9 & PMM0675-07248276 & * & 17.30 & AGN & $\mathrm{BL} \mathrm{LAC}$ & \\
\hline RX J0935.5-2802 & B & 0.23 & 9 & 35 & 31.28 & -28 & 2 & 54.0 & GSC0660900298 & 12.08 & * & $\mathrm{AC}$ & MOVe & \\
\hline RX J1023.9-4336 & B & 0.35 & 10 & 23 & 56.11 & -43 & 36 & 2.5 & PMM0450-11341838 & * & 17.50 & AGN & BL LAC & \\
\hline RX J1155.4-5641 & B & 0.27 & 11 & 55 & 27.03 & -56 & 41 & 53.3 & PMM0300-14373754 & * & 13.60 & $\mathrm{CV}$ & & $\mathrm{Y}$ \\
\hline RX J1606.6-4618 & $\mathrm{X}$ & 0.18 & 16 & 6 & 36.75 & -46 & 18 & 26.6 & GSC0831000256 & 12.03 & $*$ & $\mathrm{AC}$ & $\mathrm{G}-\mathrm{K}$ & \\
\hline RX J1639.7-3920 & $\mathrm{X}$ & 0.83 & 16 & 39 & 47.29 & -39 & 20 & 40.3 & GSC0785402093 & 13.29 & $*$ & $\mathrm{AC}$ & MOVe & \\
\hline RX J1704.3-4020 & $\mathrm{X}$ & 1.32 & 17 & 4 & 17.93 & -40 & 19 & 50.2 & HD 322763 & 10.60 & * & $\mathrm{AC}$ & A3 & \\
\hline RX J1718.4-4029 & $\mathrm{X}$ & * & * & * & * & * & * & * & $? ?$ & * & * & UNID & & $\mathrm{Y}$ \\
\hline RX J1739.4-2942 & $\mathrm{X}$ & 0.16 & 17 & 39 & 30.30 & -29 & 42 & 8.9 & A & * & $*$ & $\mathrm{Be} / \mathrm{X}$ & $\mathrm{Be}$ & $\mathrm{Y}$ \\
\hline RX J1740.7-2818 & $\mathrm{X}$ & * & * & * & * & $*$ & * & * & ?? & * & * & UNID & & $\mathrm{Y}$ \\
\hline RX J1741.4+0348 & $\mathrm{X}$ & 0.67 & 17 & 41 & 28.26 & 3 & 48 & 52.9 & PMM0900-10385820 & * & 15.30 & AGN & Sy 1 & \\
\hline RX J1742.3-2737 & $\mathrm{X}$ & * & * & * & * & $*$ & * & * & ?? & * & * & UNID & & $\mathrm{Y}$ \\
\hline RX J1749.8-3312 & $\mathrm{X}$ & * & * & * & * & $*$ & * & * & SLX 1746-331 & * & * & UNID & & $\mathrm{Y}$ \\
\hline
\end{tabular}


Table 5. continued

\begin{tabular}{|c|c|c|c|c|c|c|c|c|c|c|c|c|c|c|}
\hline Source & Sel & $d(x-o)$ & & ght & isce. & & $\overline{\mathrm{De}}$ & & Optical & $\bar{V}$ & $B$ & Class & Type & Finding \\
\hline Name & & $\left(\mathrm{r}_{90}\right)$ & $\mathrm{h}$ & $\mathrm{m}$ & $\mathrm{s}$ & d & r & 11 & Identification & & & & & Chart \\
\hline RX J1804.1+0042 & $\mathrm{X}$ & 0.56 & 18 & $\overline{4}$ & 9.01 & $\overline{0}$ & 42 & 22.1 & PMM0900-11515260 & & 18.60 & $\mathrm{Gal}$ & & \\
\hline RX J1925.0+4429 & A & 0.34 & 19 & 25 & 1.98 & 44 & 29 & 50.2 & GSC0314600035 & 9.71 & * & $\mathrm{AC}$ & $\mathrm{K} 1 \mathrm{~V}$ & \\
\hline RX J1929.8+4622 & A & 0.38 & 19 & 29 & 50.49 & 46 & 22 & 23.6 & PMM1350-10836797 & * & 17.00 & AGN & Sy 1 & \\
\hline RX J1931.1+0937 & $\mathrm{X}$ & 0.39 & 19 & 31 & 9.23 & 9 & 37 & 16.4 & A & * & 19.00 & AGN & Bl Lac & $\mathrm{Y}$ \\
\hline RX J1935.4+3746 & A & 0.02 & 19 & 35 & 29.40 & 37 & 46 & 9.9 & GSC0313500052 & 11.41 & * & $\mathrm{AC}$ & M4Ve & \\
\hline RX J1936.3+2632 & $\mathrm{S}$ & 0.53 & 19 & 36 & 18.73 & 26 & 32 & 56.5 & A & * & * & WD & DA & Y \\
\hline RX J1943.9+2118 & $\mathrm{X}$ & $*$ & $*$ & $*$ & $*$ & $*$ & * & $*$ & $? ?$ & $*$ & $*$ & UNID & & $\mathrm{Y}$ \\
\hline RX J1946.2-0444 & A & 0.37 & 19 & 46 & 16.42 & -4 & 44 & 55.4 & PMM0825-16960702 & * & 19.00 & $\mathrm{CV}$ & & $\mathrm{Y}$ \\
\hline RX J1947.3+3045 & $\mathrm{S}$ & 0.66 & 19 & 47 & 24.38 & 30 & 45 & 55.2 & PMM1200-13387458 & * & 17.10 & WD & DA & \\
\hline RX J1950.0+3821 & A & * & * & * & * & * & * & * & $? ?$ & * & * & UNID & & Y \\
\hline RX J1951.7+3716 & A & 0.65 & 19 & 51 & 47.53 & 37 & 16 & 48.3 & PMM1200-13720959 & $*$ & 15.50 & $\mathrm{CV}$ & & Y \\
\hline RX J1953.6+5025 & $\mathrm{O}$ & 0.30 & 19 & 53 & 40.76 & 50 & 24 & 57.2 & PMM1350-11463233 & * & 15.80 & $\mathrm{AC}$ & M3Ve & \\
\hline RX J1956.7+5304 & $\mathrm{O}$ & 0.30 & 19 & 56 & 46.11 & 53 & 4 & 23.2 & GSC0393501634 & 10.34 & * & $\mathrm{AC}$ & KOV & \\
\hline RX J2002.1+5438 & B & 0.61 & 20 & 2 & 7.40 & 54 & 37 & 47.0 & HD 190398 & 8.20 & 9.40 & $\mathrm{AC}$ & G0V & \\
\hline RX J2010.5+0632 & B & 0.50 & 20 & 10 & 34.41 & 6 & 32 & 17.4 & GSC0050701588 & 12.01 & * & $\mathrm{AC}$ & $\mathrm{Me}$ & \\
\hline RX J2014.8+4501 & B & 0.72 & 20 & 14 & 49.04 & 45 & 1 & 46.4 & HD 192785 & 9.20 & 10.30 & $\mathrm{AC}$ & $\mathrm{K} 0 \mathrm{~V}$ & \\
\hline RX J2019.8+2256 & A & 0.79 & 20 & 19 & 49.23 & 22 & 56 & 35.1 & GSC0215501614 & 11.40 & * & $\mathrm{AC}$ & $\mathrm{M} 4 \mathrm{Ve}$ & \\
\hline $\mathrm{RX} \mathrm{J2021.7+5213}$ & A & 0.63 & 20 & 21 & 44.59 & 52 & 13 & 38.6 & GSC0358400565 & 11.84 & * & $\mathrm{AC}$ & G3V & \\
\hline RX J2033.4+3128 & B & 0.73 & 20 & 33 & 24.41 & 31 & 28 & 12.6 & GSC0268600876 & 10.35 & * & $\mathrm{AC}$ & G7V & \\
\hline RX J2040.3+1059 & B & 0.24 & 20 & 40 & 18.55 & 10 & 59 & 45.2 & PMM0975-19711620 & * & 16.10 & AGN & Sy 1 & \\
\hline RX J2043.9+5314 & $\mathrm{X}$ & 0.26 & 20 & 43 & 59.60 & 53 & 14 & 34.9 & PMM1425-11257915 & * & 17.50 & AGN & Sy 1 & \\
\hline RX J2044.0+2833 & B & 0.52 & 20 & 44 & 4.50 & 28 & 33 & 12.1 & PMM1125-17121513 & * & 14.70 & AGN & Sy 1 & \\
\hline RX J2102.0+3359 & $\mathrm{S}$ & $*$ & * & * & * & * & * & T & ?? UV excess object & $*$ & * & UNID & & $\mathrm{Y}$ \\
\hline RX J2102.6+4553 & A & 0.84 & 21 & 2 & 38.67 & 45 & 52 & 58.4 & HD 200560 & 7.68 & 8.65 & $\mathrm{AC}$ & $\mathrm{K} 3 \mathrm{~V}$ & \\
\hline RX J2104.2+2118 & $\mathrm{X}$ & $*$ & * & * & * & * & * & * & $? ?$ & * & 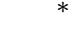 & UNID & & $\mathrm{Y}$ \\
\hline RX J2109.7+4029 & $\mathrm{X}$ & 0.87 & 21 & 9 & 48.50 & 40 & 29 & 23.9 & GSC0317201505 & 10.70 & $*$ & $\mathrm{AC}$ & $\mathrm{G} 2 \mathrm{~V}$ & \\
\hline $\mathrm{RX} \mathrm{J2133.7+5107}$ & B & 0.21 & 21 & 33 & 43.64 & 51 & 7 & 24.4 & PMM1350-14702073 & * & 15.80 & $\mathrm{CV}$ & & $\mathrm{Y}$ \\
\hline RX J2155.3+5938 & $\mathrm{S}$ & 0.69 & 21 & 55 & 23.70 & 59 & 38 & 36.6 & PMM1425-12649991 & * & 12.70 & $\mathrm{AC}$ & $\mathrm{M} 4 \mathrm{Ve}$ & \\
\hline RX J2156.3+3318 & B & 0.24 & 21 & 56 & 23.04 & 33 & 18 & 35.9 & PMM1200-18997159 & * & 16.20 & Gal & & \\
\hline RX J2255.0+5540 & $\mathrm{X}$ & 0.44 & 22 & 55 & 4.57 & 55 & 40 & 52.5 & GSC0398901784 & 9.42 & * & $\mathrm{AC}$ & G0V & \\
\hline RX J2322.6+6113 & $\mathrm{X}$ & 0.38 & 23 & 22 & 40.08 & 61 & 13 & 33.5 & GSC0427901821 & 9.79 & * & $\mathrm{AC}$ & G0V & \\
\hline
\end{tabular}

